\title{
Onset patterns in a simple model of localized parametric forcing
}

\author{
J. Porter, ${ }^{*}$ I. Tinao, A. Laverón-Simavilla, and J. Rodríguez \\ E.T.S.I. Aeronáuticos, Universidad Politécnica de Madrid, Plaza Cardenal Cisneros 3, 28040 Madrid, Spain
}

(Received 16 March 2013; published 21 October 2013)

\begin{abstract}
We investigate pattern selection at onset in a parametrically and inhomogeneously forced partial differential equation obtained by generalizing Mathieu's equation to include spatial interactions. No separation of scales is assumed. The proposed model is directly relevant to the case of parametrically forced surface waves, such as cross-waves, excited by the horizontal vibration of a fluid, where the forcing is localized to a finite region near the endwall or wavemaker. The availability of analytical solutions in the limit of piecewise constant forcing allows us investigate in detail the dependence of selected eigenfunctions on spatial detuning, forcing width, damping, boundary conditions, and container size. A wide range of onset patterns are located and described, many of which are rotated, modulated, or both, and deviate far from simple crosswise oriented standing waves. The linear selection mechanisms governing this multiplicity of potential onset patterns are discussed.
\end{abstract}

DOI: 10.1103/PhysRevE.88.042913

PACS number(s): 89.75.Kd, 47.54.-r, 47.35.-i

\section{INTRODUCTION}

A wide range of physical systems can be effectively treated as oscillators or collections of oscillators (normal modes). When small amplitude periodic forcing is applied to such systems, their response is dominated by temporal resonances where the frequency of the forcing is rationally related to one of the eigenfrequencies $\Omega$ of the unforced system. The nature of this resonant response depends on whether the forcing mechanism acts directly or parametrically. In the case of direct forcing, excitation is immediate and typically most forceful near a 1:1 resonance when the forcing frequency is equal to a natural frequency of the system. In the case of parametric forcing, on the other hand, external driving does not immediately trigger a response. The basic unexcited state persists without loss of stability until a critical forcing value is reached. In contrast to the case of direct forcing, the dominant parametric resonance is usually $1: 2$, occurring when the forcing frequency is equal to twice a natural frequency of the system.

The classical example of parametric resonance is a pendulum with vertically oscillating support. In the reference frame of the moving support, the forcing takes the form of a variable gravitational acceleration and the linear response of the system is described by Mathieu's equation [1],

$$
\ddot{A}+\left[\Omega^{2}+2 f \cos (2 t)\right] A=0 .
$$

Here the overdot denotes differentiation with respect to time, $\Omega>0$ is the natural frequency of the oscillator, and $f$ measures the amplitude of the applied forcing. Nontrivial solutions of this linear equation are located in the $(\Omega, f)$ parameter space along resonance tongues associated with integer values of $\Omega$. The widest resonance tongue and, if a typical damping term proportional to $\dot{A}$ is added, for example, the one containing the global minimum, is associated with $\Omega=1$, the subharmonic 1:2 resonance.

The Mathieu equation (1) is the prototypical model of a parametrically forced oscillator and, therefore, arises repeatedly in the study of more complex parametrically forced

*jeff.porter@upm.es systems including large pattern-forming systems like the Faraday experiment. In the standard Faraday system [2,3], a vertically vibrated container of fluid produces surface waves when driven beyond a critical value. In a reference frame attached to the container, the surface modes experience a variable gravitational force, just like the vertically vibrated pendulum. Indeed, Benjamin and Ursell [4] showed that in the inviscid, infinite depth limit the linearized Faraday problem reduces to a system of Mathieu equations. The resonance structure predicted by Eq. (1) persists even if small damping is included [5].

Although a damped version of Eq. (1), with $\Omega$ appropriately defined by the dispersion relation, can be used to predict onset values for the different eigenmodes of a large homogeneously forced system, it does not account for pattern formation in any other sense. There is no coupling between neighboring modes, and spatial modulation is ignored. Equation (1) can be generalized by including nonlinear terms, and by allowing the amplitude $A$ to depend on position and replacing $\Omega^{2}$ with a differential operator. Such generalizations, including (one-dimensional) spatial coupling, weak damping, and cubic nonlinearity, were used by Newman et al. [6] to describe a lattice of coupled vertically forced pendulums and Faraday waves in a channel. Armbruster et al. [7,8] studied an analogous equation (under different scaling assumptions) as a general model for pattern formation due to parametric resonance. We adopt a similar spirit here and generalize the basic Mathieu equation (1) to include damping and to allow spatial modulations. What qualitatively distinguishes the model proposed here from those mentioned above are (1) we consider extended two-dimensional patterns and (2) we allow spatially inhomogeneous forcing.

The generalization to spatially inhomogeneous forcing is directly motivated by recent cross-wave experiments in a horizontally vibrated container [9] where obliquely oriented patterns were observed; see also Refs. [10,11]. The parametric forcing mechanism responsible for subharmonic cross-waves, which is greatest near the endwalls or wavemaker, is not uniform and cannot be described by a generalization of Eq. (1) with constant forcing. An appropriate model must take account of the localized nature of the forcing mechanism and, in the case of a finite container, the opposite temporal phase 
of the forcing at opposing ends. The complexity introduced by inhomogeneous forcing of this type is manifest in the linear stability problem and in the variety of eigenfunctions that may describe excited patterns at onset. In contrast to typical homogeneous, isotropic extended systems, there is a complex and subtle "selection" process already at the linear level. Nonlinear processes are, of course, essential for limiting the growth of linearly unstable solutions and, via resonant interactions, for example, can be expected to contribute still more complexity to the selection of asymptotically stable patterns, especially when multiple instabilities occur close together. Such considerations are postponed for future work so that the nontrivial selection of unstable patterns by the linear problem can be fully examined. Comparison with experimental patterns (oblique cross-waves [9]) suggests that this linear selection is the key to explaining the observed patterns.

The specific results presented here follow from the application of the linearized version of the generalized Mathieu-type equation with nonuniform forcing to the case of localized parametric forcing, which is relevant to cross-waves. The availability of analytical solutions under additional simplifying assumptions allows us to directly investigate the dependence of selected onset patterns on spatial detuning, forcing width, damping, boundary conditions, and container size, all of which are difficult to vary systematically in an experiment. Other cross-wave descriptions, like the nonlinear Schrödinger equation (NLS) models [12-14] developed after Jones [15], whose linear version can also be solved exactly, rely on a separation of scales that is not assumed here (and not present in recent experiments [9,11]). Many of the solutions described below, in fact, deviate far from the ideal cross-waves taken as the basic state in the NLS models. We describe a full range of alternative solutions (rotated, modulated, or both) that may arise in parametrically forced systems such as a horizontally vibrated container of fluid. Additional complexity (multiplicity) arises from the finite width of the forcing mechanism, with associated wavenumber selection, something which cannot be captured on the slow spatial scale of the NLS models.

The paper is organized as follows. In Sec. II we introduce the linear partial differential equation to be studied. In Sec. III we locate (temporally unmodulated) subharmonic solutions of this equation in the case of piecewise constant forcing and either Neumann or Dirichlet boundary conditions, and investigate their dependence on detuning, forcing width, and damping. In Sec. IV we consider the more general case of Hopf bifurcation to temporally modulated (quasiperiodic) solutions, which is necessary in the case of finite containers. Symmetry aspects are discussed in Sec. V and conclusions presented in Sec. VI.

\section{SIMPLE MODEL WITH NONUNIFORM PARAMETRIC FORCING}

We take the following partial differential equation as a simple model describing the response of a two-dimensional pattern forming system to nonuniform parametric forcing:

$$
\ddot{h}-\nabla^{2}(h+2 \gamma \dot{h})+G(\mathbf{x}, t) h+F(h, \dot{h}, \nabla h, \ldots)=0,
$$

where $h(\mathbf{x}, t): \mathcal{R}^{2} \times t \mapsto \mathcal{R}$ is a scalar field (representing surface deformation, for example), $G(\mathbf{x}, t)$ is a temporally periodic, spatially inhomogeneous forcing function, $F$ represents nonlinear terms, $\nabla^{2}=\partial_{x}^{2}+\partial_{y}^{2}$ is the two-dimensional Laplacian operator, and $\gamma$ is a dimensionless damping parameter. If no spatial dependence or nonlinear effects are considered, then Eq. (2) reduces to Mathieu's equation (1) for $G(t)=$ $\Omega^{2}+2 f \cos (2 t)$. Similar extensions of Mathieu's equation (1) describing pattern forming systems were studied in Refs. [6-8] for the case of one-dimensional patterns and spatially uniform forcing. Note that, in the absence of damping, forcing and nonlinearity ( $\gamma=G=F=0$ ), Eq. (2) is a wave equation. Traveling wave solutions take the form $\exp \{i \Omega t+i \mathbf{k} \cdot \mathbf{x}\}$ with $\Omega=k=|\mathbf{k}|$. This simple linear dispersion relation in the undamped unforced case differs from earlier models [6-8] in that the flat solution $(\boldsymbol{k}=0)$ does not oscillate $(\Omega=0)$. This feature is consistent with the gravity-capillary surface waves that we are most interested in describing, as is the wavenumber dependence of the damping term. The Zhang-Viñals equations [16], for example, which approximate the Faraday system in the limit of weak damping and large depth, can be linearized to yield a set of damped Mathieu equations describing the evolution of each mode:

$$
\ddot{h}_{k}+2 \gamma k^{2} \dot{h}_{k}+\left[G_{0} k+\Gamma_{0} k^{3}+\gamma^{2} k^{4}+4 f k \cos (2 t)\right] h_{k}=0 .
$$

Here $h_{k}$ is the amplitude of the Fourier mode with wavenumber $k, \gamma$ is a nondimensional damping, and $G_{0}$ and $\Gamma_{0}$ are gravity and capillary numbers, respectively.

The linearization (3) of the Zhang-Viñals equations motivates the choice of damping term in Eq. (2), while the simplified dispersion relation permits simple explicit expressions for the wavenumbers of critical modes. The forcing term, as in Eq. (3), will be taken to be harmonic with period $\pi$; subharmonic waves are $2 \pi$-periodic with $k \sim 1$. For the purpose of modeling subharmonic waves in containers vibrated horizontally along the $x$ axis, we may further assume that $G(\boldsymbol{x}, t)$ is independent of $y$. Specifically, we take

$$
G(\boldsymbol{x}, t)=2 f g(x) \cos (2 t),
$$

where $f$ is a scalar amplitude and $0 \leqslant g(x) \leqslant 1$ describes the $x$ dependence of the forcing. Using Eq. (4) the linearized version of Eq. (2) becomes

$$
\ddot{h}-\nabla^{2}(h+2 \gamma \dot{h})+2 f g(x) \cos (2 t) h=0 .
$$

The solutions of this linear problem are examined in the following two sections.

\section{SUBHARMONIC PATTERNS}

At critical values of $f$, Eq. (5) may possess $2 \pi$-periodic solutions. Furthermore, since uniform parametric forcing produces standing waves at onset and the forcing (4) is independent of $y$, we look for periodic solutions that are standing in the $y$ (crosswise) direction, by writing

$$
h(\boldsymbol{x}, t)=\cos \left(k_{y} y\right)\left[P(x) e^{i t}+\text { c.c. }\right]+\cdots,
$$

where c.c. denotes the complex conjugate. Higher order terms (harmonics) can be ignored if $f$ is small, which will be the case if the damping $\gamma$ is small. Note that if $P$ is constant 
(with phase $\phi$ ), then $h \propto \cos \left(k_{y} y\right) \cos (t+\phi)$, which describes an ideal crosswise standing wave pattern. Substituting the expansion (6) into Eq. (5) and collecting terms leads to the ordinary differential equation:

$$
(1+2 i \gamma) P^{\prime \prime}+\left(1-k_{y}^{2}-2 i \gamma k_{y}^{2}\right) P=f g(x) \bar{P},
$$

where the prime denotes differentiation in $x$.

If there is no forcing $(f=0)$, then Eq. (7) has both exponentially growing and decaying solutions of the form $P(x)=\exp \{\kappa x\}$ with $\kappa$ given by

$$
\kappa^{2}=k_{y}^{2}-\frac{1}{1+2 i \gamma} .
$$

In an unbounded domain, such solutions are excluded by the physical requirement that amplitudes remain finite as $x \rightarrow$ $\pm \infty$. They may arise, however, on semi-infinite or bounded domains.

Another special case occurs if the forcing is uniform throughout some region, e.g., $g(x)=1$ for $x \in\left[x_{0}, x_{1}\right]$. Solutions of the form $P(x)=a_{1} \exp \{\kappa x\}+a_{2} \exp \{\bar{\kappa} x\}$ exist in that region if the dispersion relation

$$
\kappa^{2}=k_{y}^{2}-\frac{1 \pm \sqrt{\left(1+4 \gamma^{2}\right) f^{2}-4 \gamma^{2}}}{1+4 \gamma^{2}}
$$

is satisfied. In general $\kappa^{2}$ is a complex function of $f, \gamma$, and $k_{y}$, but for a localized region of maximum forcing $(g(x)=1$ for $x \in\left[x_{0}, x_{1}\right]$ and $g(x) \leqslant 1$ elsewhere) it may be assumed to be real. This follows from comparing to the case of maximal uniform forcing ( $g=1$ for all $x$ ), which models the vertically forced Faraday system. Valid eigenfunctions in this case must be uniformly extended (no exponential growth or decay in $x$ ), which implies that $\kappa$ is purely imaginary and thus

$$
f \geqslant \frac{2 \gamma}{\sqrt{1+4 \gamma^{2}}} .
$$

Equality holds for the minimum of the neutral stability curve $f=f_{c}\left(k_{y}^{2}-\kappa^{2}\right)$. It can be assumed that the critical forcing will be greater than this lower bound, realized with uniform forcing, when the parametric forcing is reduced in amplitude or extent by a nontrivial $g(x)$. Thus, on neutral stability curves associated with localized forcing, Eq. (10) will hold in general and $\kappa^{2}$ defined by Eq. (9) is real.

\section{A. Piecewise constant forcing on a semi-infinite domain}

Equation (7) must, in general, be solved numerically, and its solutions will depend both on $g(x)$ and on the choice of boundary conditions. However, the availability of analytical expressions for $P(x)$ when the forcing is absent or uniform means that the case of step function forcing can be especially instructive. It serves as a simplified model of the type of localized parametric forcing that is concentrated near the boundaries of a horizontally forced Faraday system. with

We first consider Eq. (7) on a semi-infinite domain $x \geqslant 0$

$$
g(x)=\left\{\begin{array}{ll}
1, & 0 \leqslant x \leqslant d \\
0, & d<x<\infty
\end{array} .\right.
$$

The boundary condition at $x=0$ is taken to be either of Neumann or Dirichlet type, while $P(x)$ is required to vanish as $x \rightarrow \infty$. These conditions pertain to the case of subharmonic waves generated near a wall or wavemaker that is far from any other wave sources.

\section{Neumann boundary conditions}

The Neumann boundary condition $P^{\prime}(0)=0$ at the wall is satisfied by the function

$$
P^{\mathrm{I}}(x)=z_{1} \cosh \left(\kappa_{1} x\right)+z_{2} \cosh \left(\kappa_{2} x\right) .
$$

This expression satisfies Eq. (7) in the forced region $0 \leqslant x \leqslant d$ (referred to as region I) provided that $\kappa_{1}^{2}$ and $\kappa_{2}^{2}$ satisfy Eq. (9); for concreteness, $\kappa_{1}\left(\kappa_{2}\right)$ will be associated with the plus (minus) sign. In the unforced region $d \leqslant x<\infty$ (region II) the solution of Eq. (7) is a simple exponential

$$
P^{\mathrm{II}}(x)=z_{3} e^{\kappa_{3}(d-x)},
$$

where $\kappa_{3}$ is the root of Eq. (8) with positive real part, to ensure that $P^{\mathrm{II}}(x) \rightarrow 0$ as $x \rightarrow \infty$.

At the step $x=d$ the solution and its first derivative must be continuous; the second derivative satisfies a jump condition that follows from Eq. (7). These continuity conditions imply that

$$
z_{1}=R\left(\kappa_{1}, \kappa_{2}\right) z_{3}, \quad z_{2}=R\left(\kappa_{2}, \kappa_{1}\right) z_{3},
$$

where

$$
R\left(\kappa_{1}, \kappa_{2}\right)=\frac{\operatorname{sech}\left(\kappa_{1} d\right)\left[\kappa_{3}+\kappa_{2} \tanh \left(\kappa_{2} d\right)\right]}{\kappa_{2} \tanh \left(\kappa_{2} d\right)-\kappa_{1} \tanh \left(\kappa_{1} d\right)} .
$$

Substituting $P^{\mathrm{I}}(x)$ into Eq. (7) leads to

$$
z_{1}=\Phi_{+} \bar{z}_{1}, \quad z_{2}=\Phi_{-} \bar{z}_{2},
$$

where

$$
\Phi_{ \pm}=\frac{(2 i \gamma-1) f}{2 i \gamma \pm \sqrt{\left(1+4 \gamma^{2}\right) f^{2}-4 \gamma^{2}}} .
$$

Note that $\left|\Phi_{ \pm}\right|=1$. Together, Eqs. (14) and (16) require $\bar{R}\left(\kappa_{1}, \kappa_{2}\right) R\left(\kappa_{2}, \kappa_{1}\right) \Phi_{+}=R\left(\kappa_{1}, \kappa_{2}\right) \bar{R}\left(\kappa_{2}, \kappa_{1}\right) \Phi_{-}$and thus

$$
\begin{aligned}
0= & 4 \gamma k_{3}\left[\kappa_{1} \operatorname{sh}_{1} \mathrm{ch}_{2}-\kappa_{2} \mathrm{ch}_{1} \mathrm{sh}_{2}\right] \\
& +\left(1+4 \gamma^{2}\right)\left(\kappa_{1}^{2}-\kappa_{2}^{2}\right)\left\{\left(k_{3}^{2}+\delta_{3}^{2}\right) \operatorname{ch}_{1} \mathrm{ch}_{2}\right. \\
& \left.+\delta_{3}\left[\kappa_{1} \operatorname{sh}_{1} \mathrm{ch}_{2}+\kappa_{2} \mathrm{ch}_{1} \operatorname{sh}_{2}\right]+\kappa_{1} \kappa_{2} \operatorname{sh}_{1} \operatorname{sh}_{2}\right\},
\end{aligned}
$$

where $\operatorname{ch}_{j} \equiv \cosh \left(\kappa_{j} d\right), \operatorname{sh}_{j} \equiv \sinh \left(\kappa_{j} d\right), j=1,2$, and $\kappa_{j}=$ $\delta_{j}+i k_{j}, j=1,2,3$ with $k_{j}, \delta_{j} \in \mathbb{R}$. Since $\kappa_{1}$ and $\kappa_{2}$ depend through Eq. (9) on $f$, Eq. (18) defines the critical forcing $f_{c}\left(k_{y}, d, \gamma\right)$ at which the solution defined by Eqs. (12) and (13) exists. Note that Eq. (18) is satisfied for any width $d$ if $\kappa_{1}=\kappa_{2}$, which corresponds to the homogeneous forcing limit and an equality relation in Eq. (10). This spurious solution must be excluded from the localized forcing case with finite damping, as expansion (12) is not valid when $\kappa_{1}=\kappa_{2} ; R(\kappa, \kappa)$ is divergent and the continuity conditions are not satisfied.

Using Eqs. (6), (12), and (13) we see that the reconstructed critical solution takes the form

$$
\begin{aligned}
h^{\mathrm{I}}(\boldsymbol{x}, t)= & 2 \cos \left(k_{y} y\right)\left[a_{1} \cosh \left(\kappa_{1} x\right) \cos \left(t+\phi_{1}\right)\right. \\
& \left.+a_{2} \cosh \left(\kappa_{2} x\right) \cos \left(t+\phi_{2}\right)\right], \\
h^{\mathrm{II}}(\boldsymbol{x}, t)= & 2 \cos \left(k_{y} y\right) e^{\delta_{3}(d-x)} \cos \left(t-k_{3} x+\tilde{\phi}_{3}\right),
\end{aligned}
$$

where the complex amplitudes determined by Eqs. (14) and (16) have been written as $z_{j}=a_{j} \exp \left\{i \phi_{j}\right\}$ for $j=1,2,3$ 
with $a_{j}, \phi_{j} \in \mathbb{R}, \tilde{\phi}_{3}=\phi_{3}+k_{3} d$, and $a_{3}$ has been set to unity. The pattern is thus composed of standing waves within the forced region $x \leqslant d$ and a damped traveling wave in the unforced region $x>d$.

\section{Dirichlet boundary conditions}

To satisfy $P(0)=0$ at the wall, Eq. (12) is replaced with

$$
P^{\mathrm{I}}(x)=z_{1} \sinh \left(\kappa_{1} x\right)+z_{2} \sinh \left(\kappa_{2} x\right),
$$

where $\kappa_{1}^{2}$ and $\kappa_{2}^{2}$ are once more given by Eq. (9). The solution $P^{\mathrm{II}}(x)$ in the unforced region again takes the form of Eq. (13).

The continuity conditions at $x=d$ lead to a modified version of Eqs. (14) with sinh and cosh interchanged:

$$
z_{1}=S\left(\kappa_{1}, \kappa_{2}\right) z_{3}, \quad z_{2}=S\left(\kappa_{2}, \kappa_{1}\right) z_{3}
$$

where

$$
S\left(\kappa_{1}, \kappa_{2}\right)=\frac{\operatorname{csch}\left(\kappa_{1} d\right)\left[\kappa_{3}+\kappa_{2} \operatorname{coth}\left(\kappa_{2} d\right)\right]}{\kappa_{2} \operatorname{coth}\left(\kappa_{2} d\right)-\kappa_{1} \operatorname{coth}\left(\kappa_{1} d\right)},
$$

while Eqs. (16) are replaced by

$$
\begin{array}{ll}
z_{1}=\varepsilon_{1} \Phi_{+} \bar{z}_{1}, & \varepsilon_{1}=\operatorname{sign}\left(\kappa_{1}^{2}\right), \\
z_{2}=\varepsilon_{2} \Phi_{-} \bar{z}_{2}, & \varepsilon_{2}=\operatorname{sign}\left(\kappa_{2}^{2}\right),
\end{array}
$$

with $\Phi_{ \pm}$defined in Eq. (17). Together, Eqs. (21) and (23) determine $f_{c}\left(k_{y} ; d, \gamma\right)$ by requiring

$$
\begin{aligned}
0= & 4 \gamma k_{3}\left[\kappa_{1} \mathrm{ch}_{1} \mathrm{sh}_{2}-\kappa_{2} \mathrm{sh}_{1} \mathrm{ch}_{2}\right] \\
& +\left(1+4 \gamma^{2}\right)\left(\kappa_{1}^{2}-\kappa_{2}^{2}\right)\left\{\left(k_{3}^{2}+\delta_{3}^{2}\right) \mathrm{sh}_{1} \mathrm{sh}_{2}\right. \\
& \left.+\delta_{3}\left[\kappa_{1} \mathrm{ch}_{1} \mathrm{sh}_{2}+\kappa_{2} \mathrm{sh}_{1} \mathrm{ch}_{2}\right]+\kappa_{1} \kappa_{2} \mathrm{ch}_{1} \mathrm{ch}_{2}\right\},
\end{aligned}
$$

which can be obtained from Eq. (18) by interchanging sinh and cosh. The degenerate solution of Eq. (24) when $\kappa_{1}=\kappa_{2}$ must again be excluded, as with Eq. (18). In addition, there is a second spurious solution on the curve $f^{2}=\left(1-k_{y}^{2}\right)^{2}+4 \gamma^{2} k_{y}^{4}$ where $\kappa_{2}=0$ when $k_{y}^{2}<1 /\left(1+4 \gamma^{2}\right)$ and $\kappa_{1}=0$ when $k_{y}^{2}>$ $1 /\left(1+4 \gamma^{2}\right)$.

Using Eqs. (6), (20), and (13) the reconstructed critical solution can be written

$$
\begin{aligned}
h^{\mathrm{I}}(\boldsymbol{x}, t)= & 2 \cos \left(k_{y} y\right)\left[\tilde{a}_{1} \sinh \left(\kappa_{1} x\right) \cos \left(t+\tilde{\phi}_{1}\right)\right. \\
& \left.+\tilde{a}_{2} \sinh \left(\kappa_{2} x\right) \cos \left(t+\tilde{\phi}_{2}\right)\right], \\
h^{\mathrm{II}}(\boldsymbol{x}, t)= & 2 \cos \left(k_{y} y\right) e^{-\delta_{3}(x-d)} \cos \left(t-k_{3} x+\tilde{\phi}_{3}\right),
\end{aligned}
$$

where

$$
\tilde{a}_{j}=\sqrt{\varepsilon_{j}} a_{j}, \quad \tilde{\phi}_{j}=\phi_{j}+\frac{\pi}{4}\left(\varepsilon_{j}-1\right), \quad j=1,2,
$$

and the three complex amplitudes $a_{j} \exp \left\{i \phi_{j}\right\}$ are determined by Eqs. (21) and (23). Again, the solution is shown to be a combination of standing waves within the forced region $x \leqslant d$ and a damped traveling wave in the unforced region $x>d$.

\section{B. Results for semi-infinite domain}

We describe here the properties of the subharmonic solutions obtained analytically in the previous section for the case of piecewise constant forcing. These depend on the crosswise wavenumber $k_{y}$, the damping $\gamma$, and the forcing width $d$.

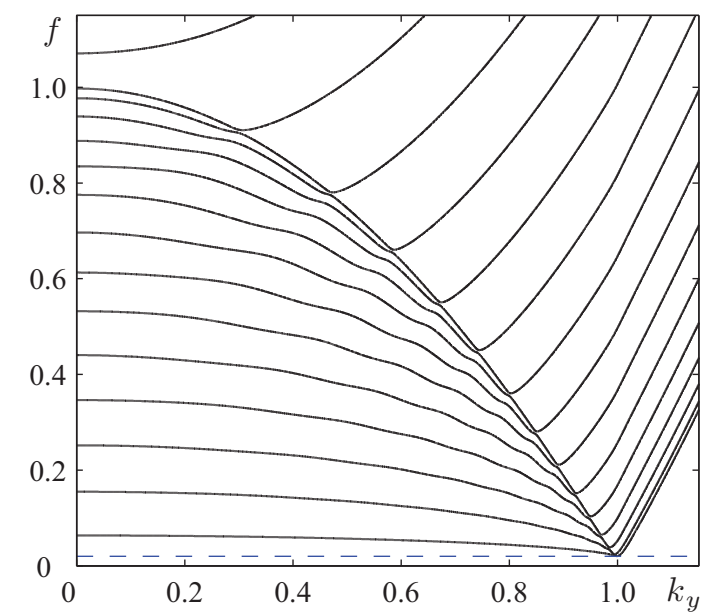

FIG. 1. (Color online) Neutral stability curves as a function of $k_{y}$ with Neumann boundary conditions, $d=10 \pi$, and $\gamma=0.01$. The dashed horizontal line marks the critical value in the uniformly forced case $(d=\infty)$. Note that, despite near-tangencies that give the appearance of intersection on this scale, neutral stability curves are isolated and do not cross.

\section{Effect of detuning}

The subharmonic solutions obtained in Sec. III depend strongly on the parameter $k_{y}$, which may be thought of as a measure of spatial detuning on a laterally confined domain where $k_{y}$ takes only discrete values. Generally speaking, subharmonic patterns are easiest to excite when $k_{y}$ is close to unity (i.e., when $k_{y}$ satisfies the inviscid dispersion relation), and this can be understood as a preference for crosswise orientation. It is possible, however to excite patterns with any value of $k_{y}$, as illustrated in Fig. 1 in the case of Neumann boundary conditions, $\gamma=0.01$, and $d=10 \pi$. The critical curves $f=f_{c}\left(k_{y}\right)$ shown in this figure organize into a series of nested tongues that do not cross. Tongues in the upper $(f>1)$ unbounded set increase monotonically with $k_{y}$ while those in the lower, finite set exhibit minima near the curve $f^{2}=\left(1-k_{y}^{2}\right)^{2}+4 \gamma^{2} k_{y}^{4}$, which is where $\kappa_{2}=0$, according to Eq. (9); if $k_{y}^{2} \geqslant 1 /\left(1+4 \gamma^{2}\right)$, then $\kappa_{1}=0$ on this curve. Although it is not plotted directly, the influence of this curve is evident in Fig. 1. Each of the lower set of neutral stability tongues not only reaches a minimum forcing value when this criteria $\left(\kappa_{2}=0\right)$ is nearly satisfied, but also continues to shadow this curve leftward before veering off just as the next minima, belonging to the neutral stability tongue immediately above, is approached. On the scale of Fig. 1 this close proximity gives the appearance of intersection, but this does not occur. The solution for a given set of parameters is unique when it exists. As shown below, the nested neutral stability tongues can be thought of as the locus of solutions with increasing (half) oscillations in the forced region. The number of tongues with local minima at finite $k_{y}$ increases with width $d$ and reduces to one for sufficiently narrow forcing.

Several of the lowest instability tongues in Fig. 1 are shown in more detail in Fig. 2, which also shows the pattern associated with the minimum of the lowest tongue. Note that, although this minimum occurs at $k_{y} \simeq 1$ and the pattern is reminiscent of cross-waves, it is clearly modulated in the $x$ direction as well. 


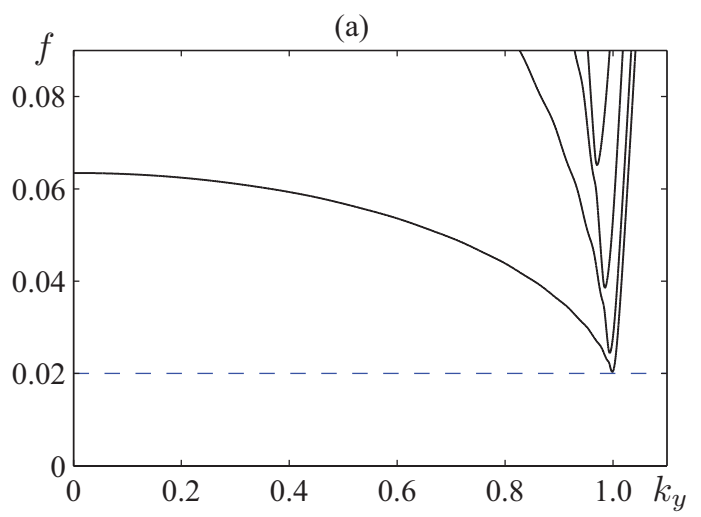

(b)

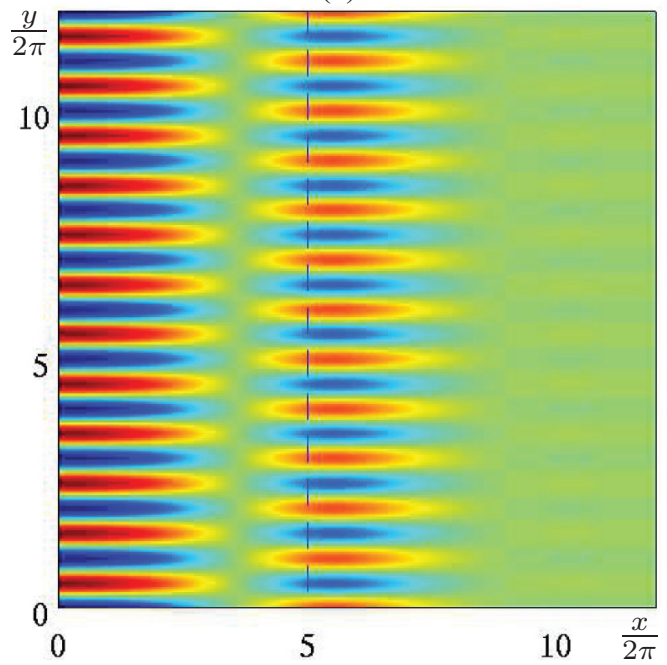

FIG. 2. (Color online) (a) Neutral stability curves as a function of $k_{y}$ with Neumann boundary conditions, $d=10 \pi$, and $\gamma=0.01$. (b) Pattern corresponding to the minimum of the lowest tongue at $k_{y}=0.9988, f_{c}=0.02027$ for $t=0.9$ (chosen for aesthetic reasons). The dashed horizontal line in (a) marks the critical forcing in the uniform $(d=\infty)$ case, while the vertical dashed line in (b) marks the edge of the forced region.

The effect of detuning $\left(k_{y}\right)$ on modulation in the $x$ direction can be seen in Fig. 3, which shows $k_{1}=\operatorname{Im}\left(\kappa_{1}\right)$ and $k_{2}=\operatorname{Im}\left(\kappa_{2}\right)$ over the range $0<k_{y}<1.2$ for the four lowest instability tongues. Note that, especially for the lowest mode, $k_{1}$ and $k_{2}$ stay close to the curve $\sqrt{(}\left(1-k_{y}^{2}\right)$, which shows the influence of the dispersion relation. However, there is an increasing split between $k_{1}$ (solid curves) and $k_{2}$ (dashed curves) for successively higher instability tongues. Observe that for most values of $k_{y}<1$ both components of the forced solution are oscillatory. However, just prior to reaching $k_{y}=1$ the $\kappa_{2}$ root reaches the origin and moves onto the real axis, signaling that the corresponding solution component becomes exponential in nature. The remaining oscillatory root $\kappa_{1}=i k_{1}$ then satisfies the condition $k_{1} d \simeq(n-1 / 2) \pi$ with increasing accuracy as $k_{y}$ increases; here $n=1,2, \ldots$ enumerates the neutral stability curves from the lowest tongue upward. This suggests that for positive detuning $\left(k_{y}>1\right)$ the solution has an approximate node at $x=d$ and is small outside of the forced region. This picture is confirmed in Fig. 4, which shows the quantities $a_{j} \cosh \left(\kappa_{j} d\right)$ characterizing the amplitude of each

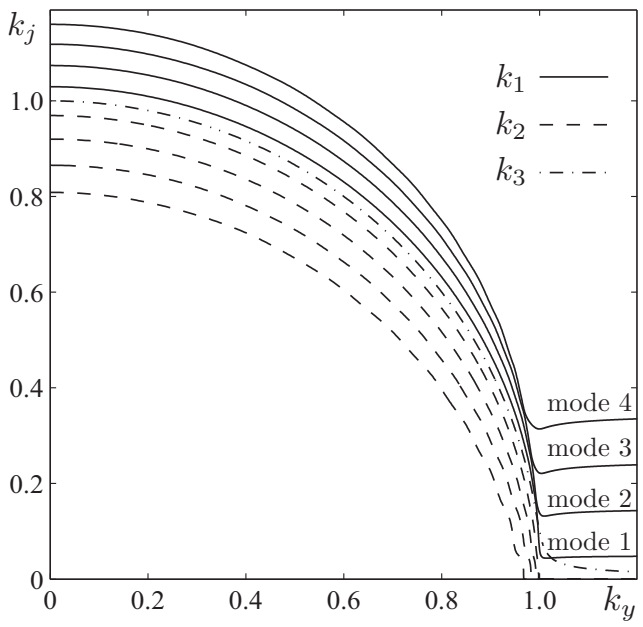

FIG. 3. Modulation wavenumbers $k_{1}$ (solid curves), $k_{2}$ (dashed curves), and $k_{3}$ (dash-dotted curve) for the first four instability tongues as a function of $k_{y}$ with Neumann boundary conditions, $d=10 \pi$, and $\gamma=0.01$. The splitting between $k_{1}$ and $k_{2}$ increases with successive modes (successively higher instability tongues).

solution component at the edge of the forced region (temporal phase is ignored). Note that both components are comparable for $k_{y}<1$, when the solution in the forced region is completely oscillatory, but after $\kappa_{2}$ becomes real, this second component becomes increasingly inconsequential, and the solution within the forced region approaches a simple cosine function with wavenumber $k_{1}$. The exponentially growing component plays little role beyond $k_{y} \simeq 1$.

The dramatic effect of detuning near $k_{y}=1$ is further illustrated in Fig. 5. This figure shows $\operatorname{Re}(P(x))$, the $x$ profile of the pattern at $t=0$, for the lowest instability tongue as well as $a_{1} \cosh \left(\kappa_{1} x\right) \cos \left(\phi_{1}\right)$ and $a_{2} \cosh \left(\kappa_{2} x\right) \cos \left(\phi_{2}\right)$, the two components in the forced region. Observe that for $k_{y}=0.95$ the pattern is strongly oscillatory in $x$ and extends far into the unforced region. For $k_{y}=1.05$ the $\kappa_{2}$ component is negligible, and the modulation resembles a cosine function (oscillating through nearly a quarter period) with a small exponential tail extending into in the unforced region.

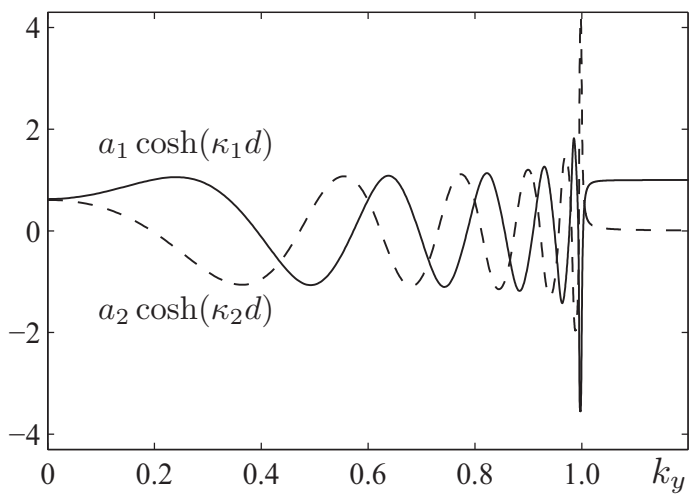

FIG. 4. The magnitude of the two solution components at the edge of the forced region, illustrated with the quantities $a_{1} \cosh \left(\kappa_{1} d\right)$ and $a_{2} \cosh \left(\kappa_{2} d\right)$, for Neumann boundary conditions, $d=10 \pi$, and $\gamma=0.01$ 


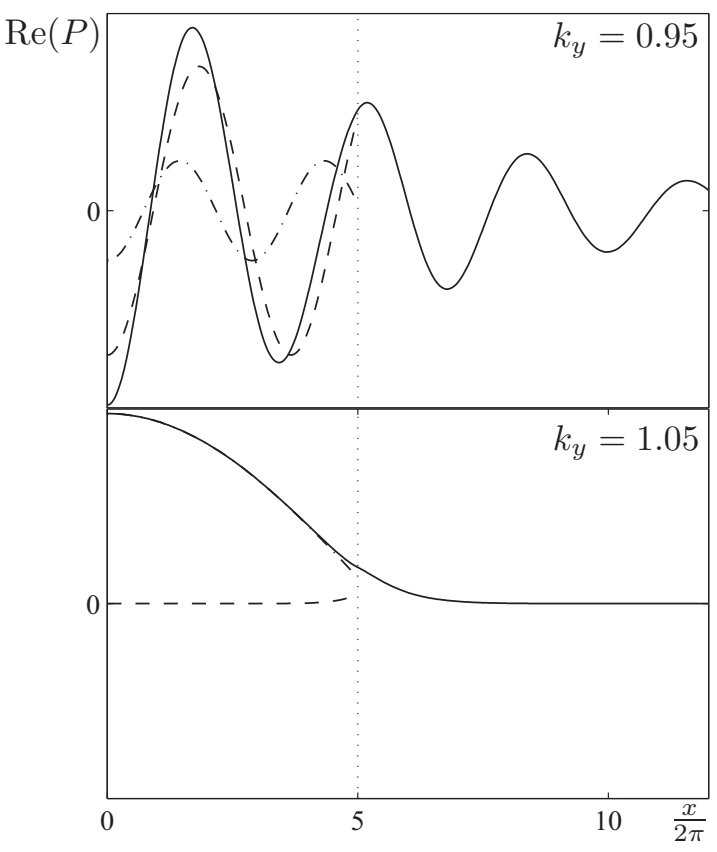

FIG. 5. Effect of detuning on pattern profile for the lowest neutral stability tongue. $\operatorname{Re}(P(x))$ is shown as a solid curve and the two components in the forced region, $a_{j} \cosh \left(\kappa_{j} x\right) \cos \left(\phi_{j}\right), j=1,2$, as dash-dotted and dashed curves, respectively. Neumann boundary conditions are used with $d=10 \pi$ and $\gamma=0.01$. The vertical dotted line marks the boundary of the forced region.

The difference between successive neutral stability tongues can be seen by comparing Fig. 5 with Fig. 6, which shows the profile of the pattern associated with the second tongue with minimum at $k_{y}=0.99404$, again for the values $k_{y}=0.95$ and $k_{y}=1.05$. Note that the profile of the pattern at $k_{y}=0.95$ is qualitatively similar to the corresponding profile for the lowest neutral stability tongue. The difference is that $\kappa_{2}$ becomes real sooner, when $k_{y}$ is a bit smaller and $k_{1}$ a bit larger, so that an additional half oscillation, approximately, is achieved in the forced region. This illustrates the approximate condition $k_{1} d \simeq(n-1 / 2) \pi, n=1,2, \ldots$, mentioned above, that holds for positive detuning $\left(k_{y}>1\right)$.

The boundary conditions at $x=0$ are not important as long as $d$ is large. When using Dirichlet boundary conditions instead of Neumann there are only minor changes in the critical curves $f_{c}\left(k_{y}\right)$, which are raised slightly, and in the pattern observed at onset, which must have a node at $x=0$ but is otherwise similar. This can be seen by comparing Fig. 7 with Fig. 2. The dependence on $k_{y}$ (detuning) is analogous except that the condition for large $k_{y}$ becomes $k_{1} d \simeq n \pi, n=1,2, \ldots$, which can be seen by comparing Fig. 8 with Fig. 3 .

\section{Effect of forcing width}

The extent of the forcing compared to the wavelength of the instability is a critical parameter in localized forcing problems. It is obvious that as the width of the forced region decreases it will become increasingly difficult to excite patterns with the mechanism of Eq. (5). Furthermore, the width of the forced region is expected to play a key role in selecting the orientation of the pattern at onset. When $d$ is very large, for example, the

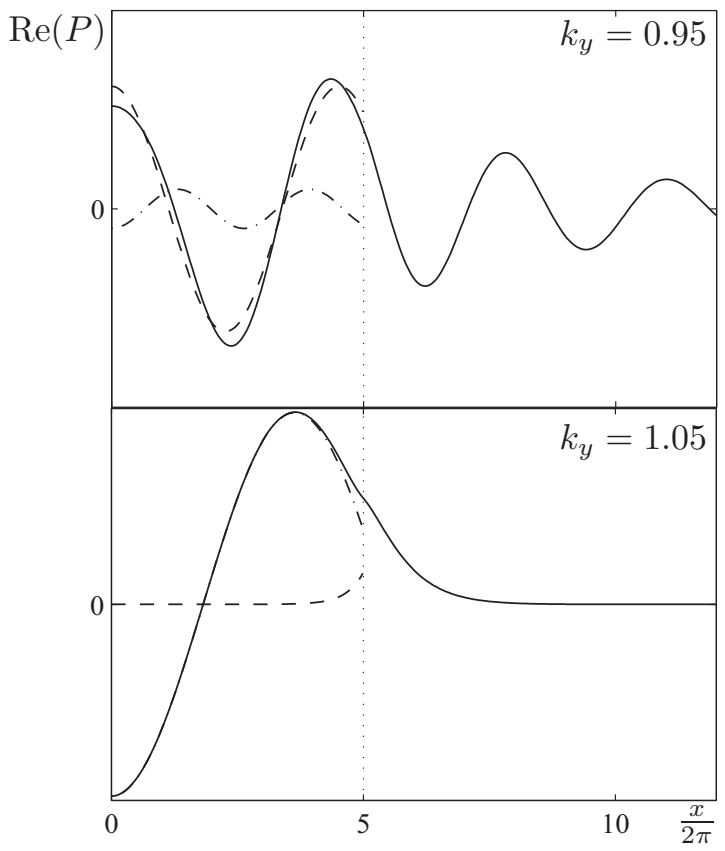

FIG. 6. Effect of detuning on pattern profile for the second neutral stability tongue. $\operatorname{Re}(P(x))$ is shown as a solid curve and the two components in the forced region, $a_{j} \cosh \left(\kappa_{j} x\right) \cos \left(\phi_{j}\right), j=1,2$, as dash-dotted and dashed curves, respectively. Neumann boundary conditions are used with $d=10 \pi$ and $\gamma=0.01$. The vertical dotted line marks the boundary of the forced region.

problem approaches the uniform homogeneously forced case where waves of any orientation may appear at onset, like in the vertically forced Faraday wave problem on an unbounded domain. A large forced region should therefore lead to a weak selection of pattern orientation at onset. When $d$ is small, on the other hand, the system should show some degree of preference for crosswise orientation, consistent with experiments using horizontally forced systems [9] or wavemakers [3,14,17-22].

First consider the case of Neumann boundary conditions with relatively narrow forcing $(d=2 \pi / 5)$ shown in Fig. 9(a). Note that the nested series of critical curves present in Fig. 2(a) is gone, although additional curves do exist at forcing values beyond those shown in the figure. As with the case of wider forcing $(d=10 \pi)$ the minimum of the lowest neutral stability tongue occurs very close to $k_{y}=1$ and is associated with a crosswise oriented pattern, modulated in the $x$ direction, as shown in Fig. 9(b). As anticipated, the selection of this pattern is stronger than with $d=10 \pi$ in the sense that it is more isolated from higher instability tongues, and its threshold is lower relative to the threshold of obliquely oriented patterns with smaller values of $k_{y}$. A pattern with $k_{y}=0$, for example, requires a forcing value of $f=0.916$ compared to the onset value of $f=0.1221$ at $k_{y}=0.999$. In the opposite limit of $d \rightarrow \infty$ the (lowest set of) neutral stability curves are asymptotic for $k_{y}<1$ to the uniform forcing value (dotted line), and the difference in critical forcing values disappears.

With Dirichlet boundary conditions and small forcing width $d$, the situation is quite different, as shown in Fig. 10. The critical forcing value is much higher than in the Neumann case, since requiring $h(0, y, t)=0$ suppresses the effect of parametric forcing near $x=0$. In addition to this delay of 


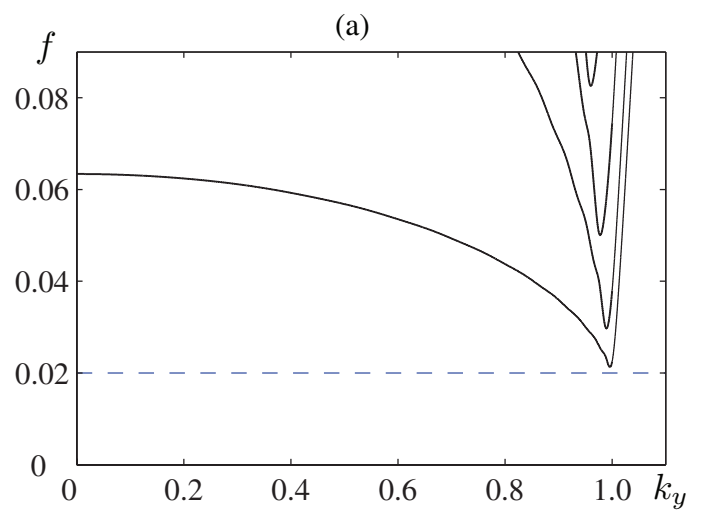

(b)

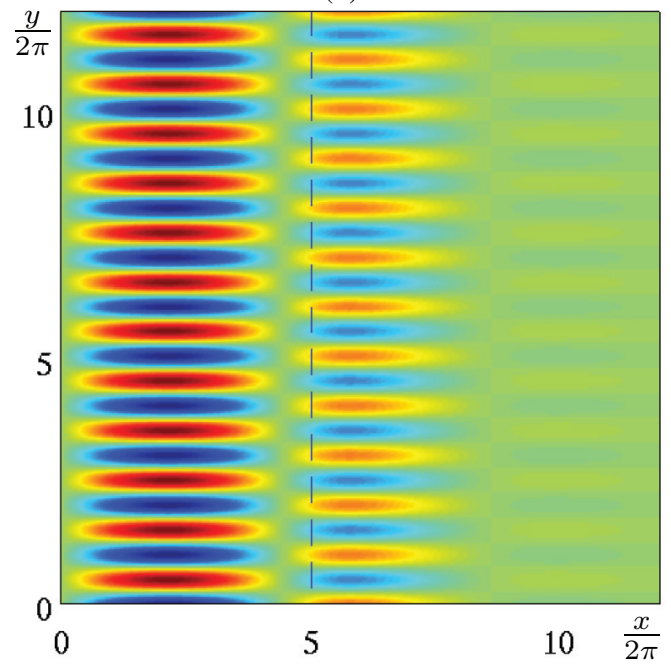

FIG. 7. (Color online) (a) Neutral stability curves as a function of $k_{y}$ with Dirichlet boundary conditions, $d=10 \pi$, and $\gamma=0.01$. (b) Pattern corresponding to the minimum of the lowest tongue at $k_{y}=0.9959$ and $f=0.02127$, for $t=1.1$. The dashed horizontal line in (a) marks the critical forcing in the uniform $(d=\infty)$ case, while the vertical dashed line in (b) marks the edge of the forced region.

onset, the location of the minima moves to a smaller value of $k_{y}=0.9326$, which leads to a compensating increase in $k_{3}$, to maintain $k_{y}^{2}+k_{3}^{2} \simeq 1$, and hence more rapid oscillations in $x$. This effect on pattern orientation of decreasing $d$ is contrary to that seen with Neumann boundary conditions. The forcing mechanism is relatively ineffective with Dirichlet boundary conditions and small $d$, and depends only weakly on $k_{y}$, meaning that the linear selection of crosswise orientation is similarly weak and, in this case, the pattern that appears at onset is a significantly rotated one.

Continuously varying $d$ over the range $0.2 \pi \leqslant d \leqslant 40 \pi$ and obtaining the solution at the minimum of the lowest instability tongue provides the data shown in Fig. 11 for the case of Neumann boundary conditions and in Fig. 12 for the case of Dirichlet boundary conditions. For either boundary condition, the critical forcing approaches the value 0.019996 of the uniformly forced case in the limit of large $d$. In the Neumann case the critical forcing $f_{c}$ increases with $\alpha=2 \pi / d$ in a nearly linear fashion, at least for $\alpha \geqslant 0.5$, while in the Dirichlet case the critical forcing value increases much faster.

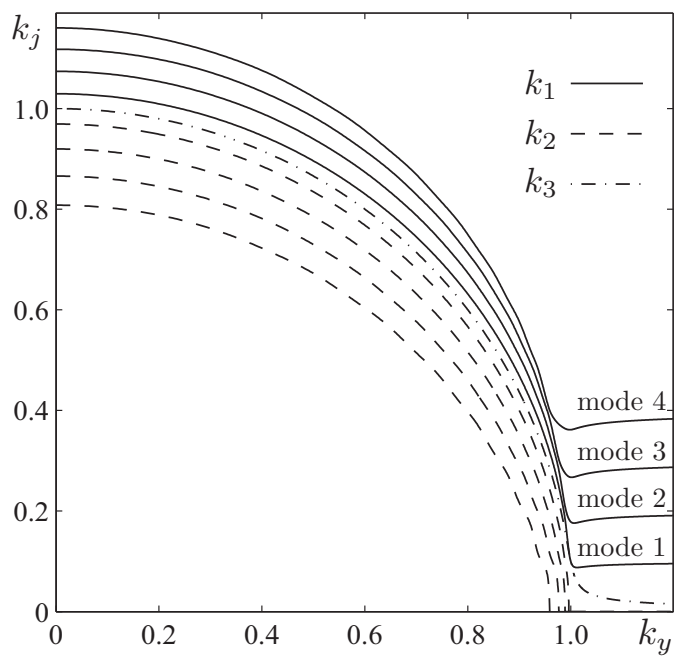

FIG. 8. Modulation wavenumbers $k_{1}$ (solid curves), $k_{2}$ (dashed curves), and $k_{3}$ (dash-dotted curve) for the first four instability tongues as a function of $k_{y}$ with Dirichlet boundary conditions, $d=10 \pi$, and $\gamma=0.01$. The splitting between $k_{1}$ and $k_{2}$ increases with successive modes (successively higher instability tongues).

The effect that varying forcing width has on the pattern wavenumbers and thus on the orientation of the selected onset pattern can be seen in Fig. 11(b) and 11(c) and Fig. 12(b) and 12(c). In the Neumann case the minimum of the lowest instability tongue occurs very close to (but always slightly less than) $k_{y}=1$ across the entire range of $d$ values. This means, according to Eq. (8), that $k_{3}$ remains small, on the order of $\sqrt{\gamma}$. Thus, outside of the forced region the onset pattern is only slightly rotated (modulated), with an apparent angle of $84.17^{\circ}$, and this can be attributed to the effect of the damping $\gamma$. There is little dependence on $d$. Within the forced region, $x \leqslant d$, the standing wave solution is the sum of an oscillatory part, $\cos \left(k_{1} x\right)$, and an exponentially growing part, $\cosh \left(\kappa_{2} x\right)$, because over this range of values the minimum occurs where $\kappa_{1}$ is imaginary and $\kappa_{2}$ is real (see Fig. 3). The oscillatory part is characterized by a wavenumber $k_{1}$ that increases monotonically with $\alpha$, leading to an ever more rotated pattern. The extent $d$ of this more rotated pattern is relatively small, however, and (for the lowest instability tongue) less than a single oscillation is achieved before the transition to a damped traveling wave in region II; see Fig. 9. In fact, a closer examination of the curve $k_{1}(\alpha)$ shows a change in behavior near $\alpha=0.5$. For smaller $\alpha$ (larger $d$ ), the wavenumber $k_{1}$ can be estimated from the approximate condition $k_{1} d \sim \pi / 2$, or $k_{1} \sim \alpha / 4$, as discussed in relation with Fig. 3. For larger $\alpha$, however, this relation no longer holds, and the increase in $k_{1}$ with $\alpha$ is slower.

With Dirichlet boundary conditions, there is a stronger dependence on forcing width, as shown in Fig. 12. Not only does the critical forcing increase much faster with $\alpha$, as evident in Fig. 12(a), but the location of the minimum varies as well. Figure 12(b) shows that, although the value of $k_{y}$ at the minimum remains relatively close to 1 , there is a noticeable decrease with increasing $\alpha$, in contrast to the case of Neumann boundary conditions in Fig. 11(b). This means that $k_{3}$ must increase in order to satisfy the dispersion relation (8) in region II, and the onset pattern becomes increasingly rotated, reaching 


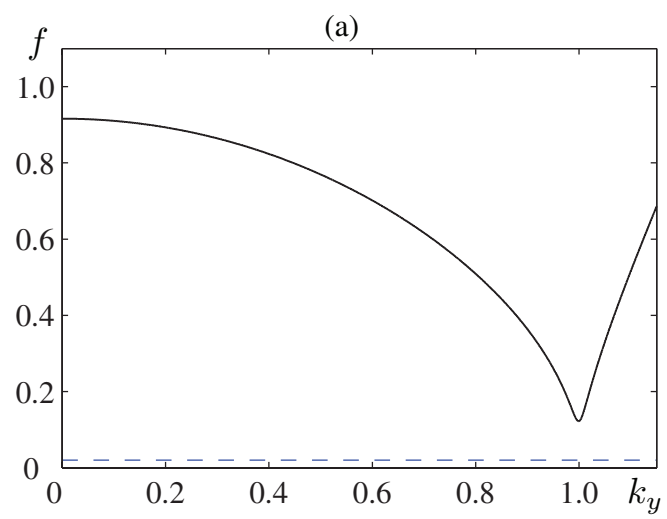

(b)

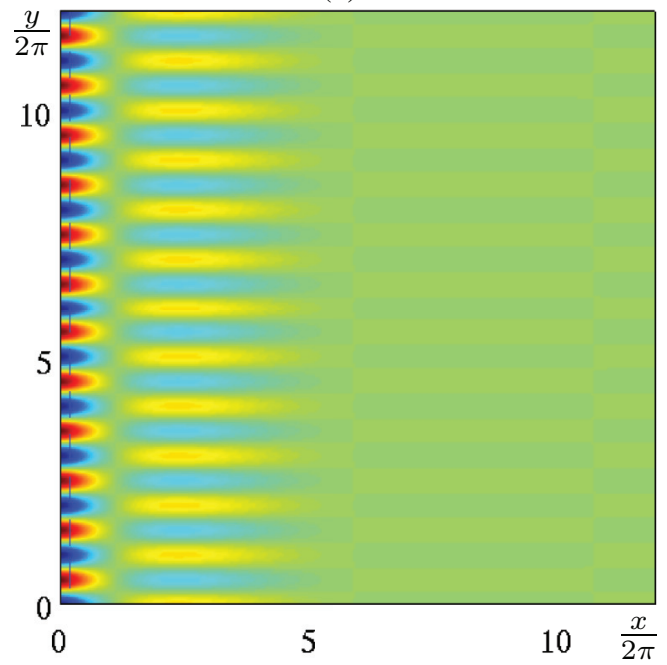

FIG. 9. (Color online) Narrow forcing width $d=0.4 \pi$ with Neumann boundary conditions and $\gamma=0.01$. (a) Lowest neutral stability curve as a function of $k_{y}$. (b) Pattern realized at the minima, $k_{y}=0.999$ and $f=0.1221$, for $t=1.1$. The dashed horizontal line in (a) marks the critical value in the uniformly forced case, while the vertical dashed line in (b) marks the edge of the forced region.

$\theta_{3}=62.9^{\circ}$ at $\alpha=10$. The wavenumber $k_{1}$ that characterizes the forced region also increases more dramatically. By $\alpha=$ 3.46, for example, $k_{1}$ has already surpassed $k_{y}$ and $\theta_{1}<45^{\circ}$. Furthermore, the root $\kappa_{2}$ is not always real in this case, but becomes imaginary for $\alpha<0.273$, which causes the small bubble seen in Fig. 12(b) and 12(c) where both components of the forced solution are oscillatory. In general, enforcing Dirichlet boundary conditions with a narrow forcing function tends to select onset patterns that are obliquely oriented. It should be recalled that this selection itself is weak, i.e., for small $d$ the (lowest) neutral stability curve is relatively flat (see Fig. 10) and onset patterns with other values of $k_{y}$ (other orientations) are only weakly damped at onset.

\section{Effect of damping}

In addition to $k_{y}$ and the profile of the forcing function $g(x)$, Eq. (7) depends on the dimensionless damping $\gamma$. Although the simplification leading to Eq. (7) loses validity for large $\gamma$, when temporal harmonics can no longer be ignored, it is possible to systematically consider the $\gamma \rightarrow 0$ limit, which is not easy to access in large-aspect-ratio experiments. (a)

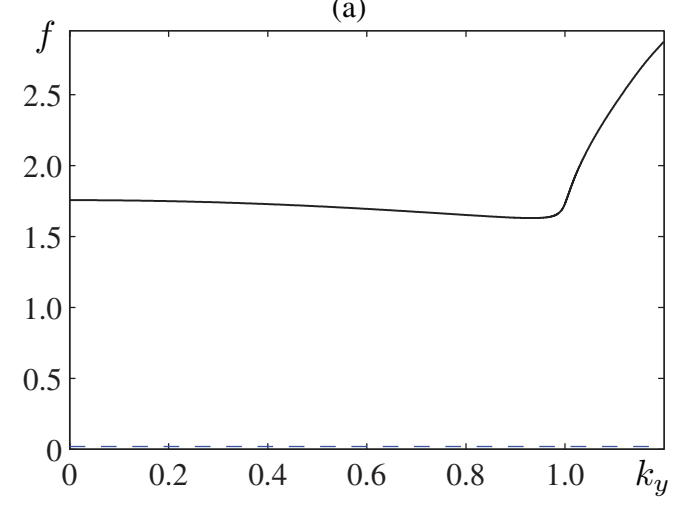

(b)

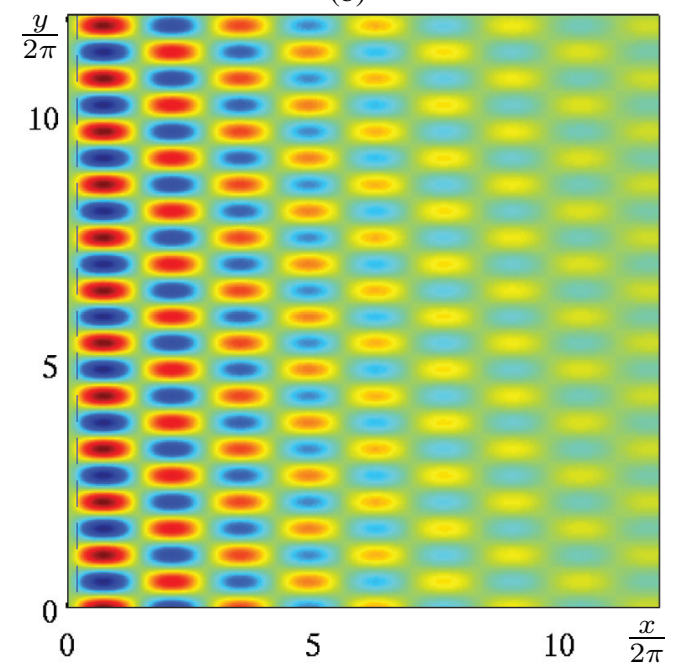

FIG. 10. (Color online) (a) Critical forcing curves as a function of $k_{y}$ with Dirichlet boundary conditions, $d=0.4 \pi$, and $\gamma=0.01$. (b) Pattern corresponding to the minima of the instability tongue at $k_{y}=0.9327, f_{c}=1.6299$, and $t=0$ The dashed horizontal line in (a) marks the critical forcing in the uniformly forced case, while the vertical dashed line in (b) marks the edge of the forced region.

Figure 13 shows how the properties of the eigenfunction at the minimum of the lowest instability tongue vary with damping for forcing width $d=2 \pi$ and Neumann boundary conditions at $x=0$. Figure 13(a) shows that, as expected, the critical forcing increases with $\gamma$ and is always slightly above the uniform homogeneous limit of Eq. (10). Figure 13(b) shows that $k_{y}$ remains very near unity until $\gamma \gtrsim 0.1$. At this point the minima in $f_{c}\left(k_{y}\right)$ moves toward smaller wavenumbers because such modes experience less damping in Eq. (2). Results for $\gamma \gtrsim 0.1$ are included for completeness but should be regarded with caution due to the errors involved in ignoring temporal harmonics. The most salient point of Fig. 13(b) and $13(\mathrm{c})$ is that viscosity is crucial in the rotation of the patterns away from pure crosswise orientation $\left(\theta_{1}, \theta_{3} \simeq 90^{\circ}\right)$. As damping decreases, the modulations in the $x$ direction become slower, and the onset pattern approaches a purely crosswise one. This damping must be quite small, however, to avoid noticeable modulation; a deviation of less than $5^{\circ}$ in $\theta_{1}$, for example, requires $\gamma<0.001$.

When Dirichlet boundary conditions apply, the eigenfunctions tend to be more rotated, with greater modulation in $x$ (see 


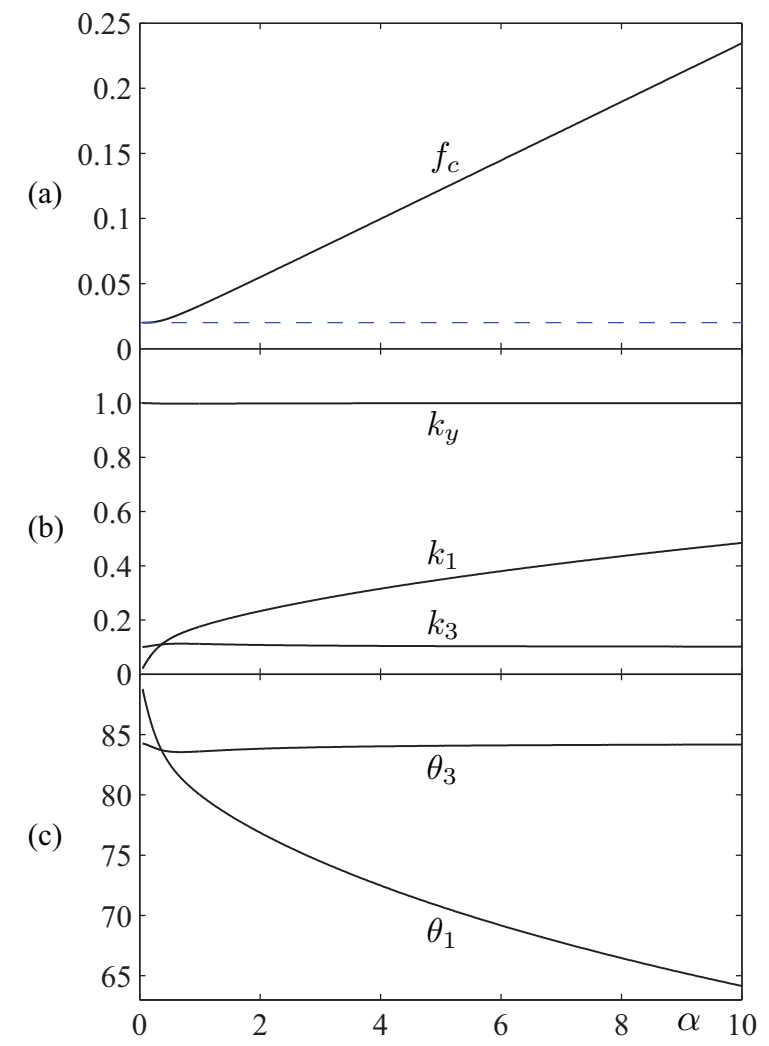

FIG. 11. (Color online) Dependence on forcing width with Neumann boundary conditions and $\gamma=0.01$. (a) Critical forcing value $f_{c}$ versus $\alpha=2 \pi / d$. The dashed horizontal line shows the critical forcing in the uniformly forced case. (b) Wavenumbers $k_{y}, k_{1}$, and $k_{3} ; k_{2}=$ 0 over this range. (c) $\theta_{1}=\arctan \left(k_{y} / k_{1}\right)$ and $\theta_{3}=\arctan \left(k_{y} / k_{3}\right)$.

Fig. 10) than with Neumann boundary conditions. Physically, this reflects the need for a rapid increase in $P(x)$, which must start from zero at the boundary $x=0$, in order to satisfy the continuity conditions at $x=d$. Figure 14 shows how the properties of the eigenfunction at the minimum of the lowest instability tongue vary with damping for $d=2 \pi$ and Dirichlet boundary conditions. In Fig. 14(a) the critical forcing can be observed above the uniform forcing value given by Eq. (10), and more so than in Fig. 13(a). Again, this shows how Dirichlet boundary conditions delay parametric instability when the width $d$ is not large. As with Neumann boundary conditions, viscosity can be seen to drive a rotation of the eigenfunction away from crosswise orientation. This is particularly clear in the behavior of $k_{3}\left(\theta_{3}\right)$, which is similar with either boundary condition. The main effect of the boundary condition is on the component of the eigenfunction within the forced region. In the limit $\gamma \rightarrow 0$ with Dirichlet boundary conditions the angle $\theta_{1}$, which describes the orientation of the $z_{1} \sin \left(k_{1} x\right)$ standing wave term in the eigenfunction of Eq. (20), approaches a value of $76^{\circ}$, not $90^{\circ}$. This limiting value depends on the width, approaching $90^{\circ}$ for large $d$. Also note that, in contrast to the Neumann case, the second root $\kappa_{2}$ becomes imaginary for $\gamma>0.147$.

\section{Antisymmetric forcing on a bounded domain}

If the domain is finite, $x \in[0, L]$, then the solutions of Eq. (5) have to satisfy an additional boundary condition at (a)

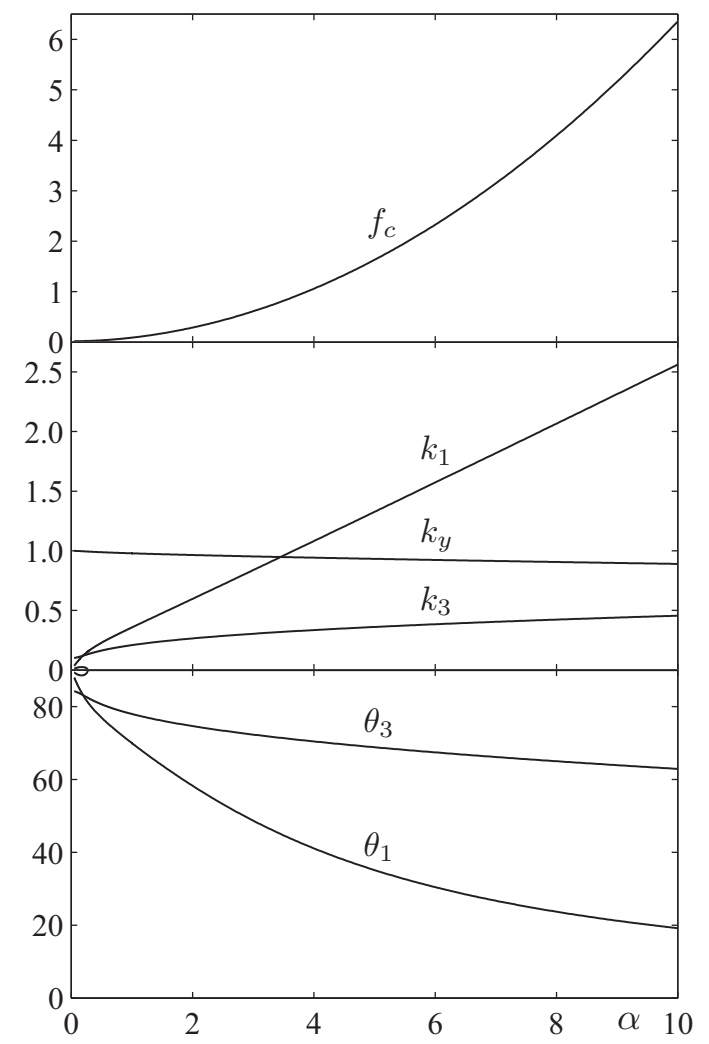

FIG. 12. Dependence on forcing width with Dirichlet boundary conditions and $\gamma=0.01$. (a) Critical forcing value $f_{c}$ versus $\alpha=$ $2 \pi / d$. (b) Wavenumbers $k_{y}, k_{1}, k_{2}$, and $k_{3}$. Note that $k_{2}$ (not labeled) is only nonzero for $\alpha<0.273$; this appears as a small bubble. (c) $\theta_{1}=\arctan \left(k_{y} / k_{1}\right), \theta_{2}=\arctan \left(k_{y} / k_{2}\right)$ (not labeled), and $\theta_{3}=\arctan \left(k_{y} / k_{3}\right)$.

$x=L$ that leads to a wavenumber selection problem in $x$. Since the main interest here is in modeling a horizontally vibrated, rigid container, we replace Eq. (11) with an antisymmetric piecewise-constant forcing function

$$
g(x)=\left\{\begin{aligned}
1, & 0 \leqslant x \leqslant d \\
0, & d<x<L-d \\
-1, & L-d \leqslant x \leqslant L
\end{aligned}\right.
$$

to reflect the fact that the motion of the wall (wavemaker) is out of phase at opposing ends. With $g(x)$ given by Eq. (27), we begin by seeking subharmonic solutions of Eq. (5) in the form of Eq. (6).

\section{Neumann boundary conditions}

The expansion (12), which satisfies $P^{\prime}(0)=0$, still applies for the uniformly forced region $0 \leqslant x \leqslant d$ (region I) and a reflected version applies for $L-d \leqslant x \leqslant L$ (region III). For $d<x<L-d$ (region II), expansion (13) must be augmented to include both growing and decaying traveling waves. Altogether, we write

$$
\begin{aligned}
p^{\mathrm{I}}(x) & =z_{1} \cosh \left(\kappa_{1} x\right)+z_{2} \cosh \left(\kappa_{2} x\right), \\
p^{\mathrm{II}}(x) & =z_{3} e^{-\kappa_{3}(x-d)}+w_{3} e^{\kappa_{3}(x-L+d)}, \\
p^{\mathrm{III}}(x) & =w_{1} \cosh \left[\kappa_{1}(L-x)\right]+w_{2} \cosh \left[\kappa_{2}(L-x)\right],
\end{aligned}
$$

where $\kappa_{1}$ and $\kappa_{2}$ satisfy Eq. (9) and $\kappa_{3}$ is given by Eq. (8). 
(a)

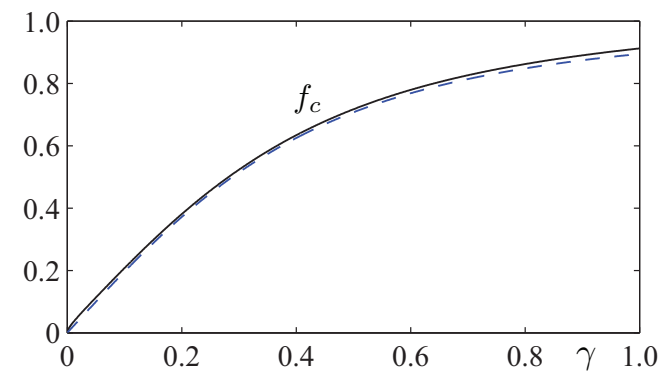

(b)

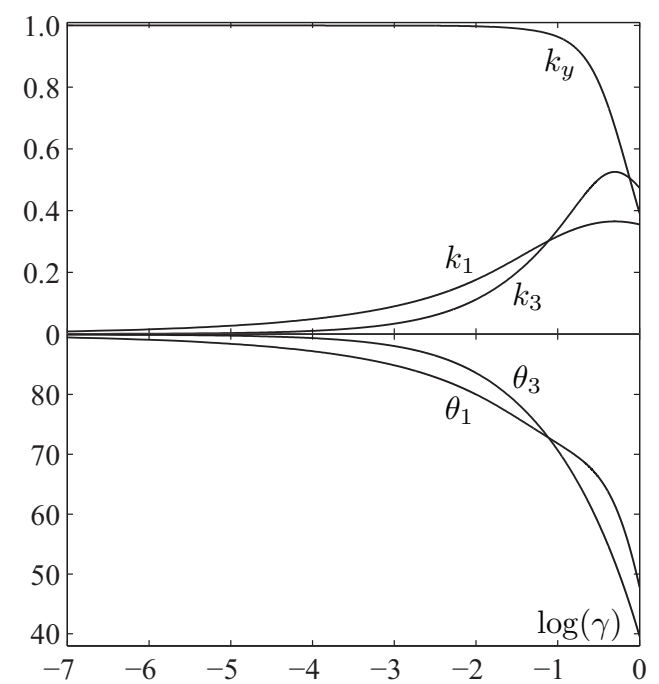

FIG. 13. (Color online) Dependence on damping with Neumann boundary conditions and $d=2 \pi$. (a) Critical forcing value versus $\gamma$. The dashed curve shows the uniform forcing limit of Eq. (10). (b) Wavenumbers $k_{y}, k_{1}$, and $k_{3} ; k_{2}$ is zero over this range of $\gamma$. (c) $\theta_{1}=\arctan \left(k_{y} / k_{1}\right)$ and $\theta_{3}=\arctan \left(k_{y} / k_{3}\right)$. Here $\log (\gamma)$ denotes the common logarithm (base 10).

Requiring continuity of $P(x)$ and $P^{\prime}(x)$ at $x=d$ and $x=$ $L-d$ yields the equations

$$
\begin{aligned}
& 0=\operatorname{ch}_{1} z_{1}+\operatorname{ch}_{2} z_{2}-z_{3}-\varepsilon_{3} w_{3}, \\
& 0=\kappa_{1} \operatorname{sh}_{1} z_{1}+\kappa_{2} \operatorname{sh}_{2} z_{2}+\kappa_{3} z_{3}-\kappa_{3} \varepsilon_{3} w_{3}, \\
& 0=\operatorname{ch}_{1} w_{1}+\operatorname{ch}_{2} w_{2}-w_{3}-\varepsilon_{3} z_{3}, \\
& 0=\kappa_{1} \operatorname{sh}_{1} w_{1}+\kappa_{2} \operatorname{sh}_{2} w_{2}+\kappa_{3} w_{3}-\kappa_{3} \varepsilon_{3} z_{3},
\end{aligned}
$$

where $\varepsilon_{3} \equiv \exp \left\{\kappa_{3}(2 d-L)\right\}$.

The four equations (29) and their complex conjugates can be used, in conjunction with Eq. (7), to obtain the linear system

$$
\left(\begin{array}{cc}
\mathrm{A}_{\infty} & \Lambda_{L} \\
\Lambda_{L} & \mathrm{~A}_{\infty}
\end{array}\right)\left(\begin{array}{c}
Z \\
W
\end{array}\right)=0
$$

where

$$
\begin{gathered}
\mathrm{A}_{\infty}=\left(\begin{array}{cccc}
\mathrm{ch}_{1} & \mathrm{ch}_{2} & -1 & 0 \\
\kappa_{1} \operatorname{sh}_{1} & \kappa_{2} \operatorname{sh}_{2} & \kappa_{3} & 0 \\
\operatorname{ch}_{1} \bar{\Phi}_{+} & \operatorname{ch}_{2} \bar{\Phi}_{-} & 0 & -1 \\
\kappa_{1} \operatorname{sh}_{1} \bar{\Phi}_{+} & \kappa_{2} \operatorname{sh}_{2} \bar{\Phi}_{-} & 0 & \bar{\kappa}_{3}
\end{array}\right), \\
\Lambda_{L}=\left(\begin{array}{cccc}
0 & 0 & -\varepsilon_{3} & 0 \\
0 & 0 & -\kappa_{3} \varepsilon_{3} & 0 \\
0 & 0 & 0 & \bar{\varepsilon}_{3} \\
0 & 0 & 0 & \bar{\kappa}_{3} \bar{\varepsilon}_{3}
\end{array}\right)
\end{gathered}
$$

(a)

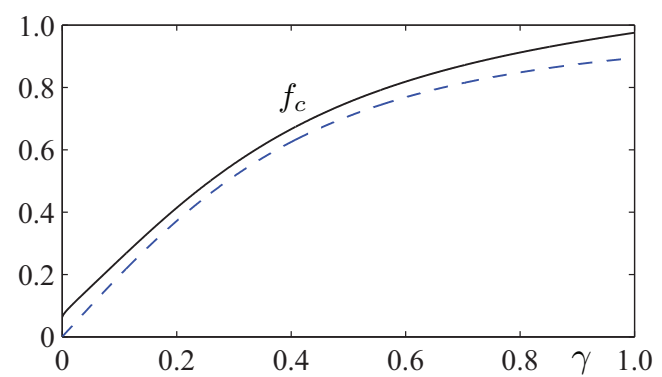

(b)

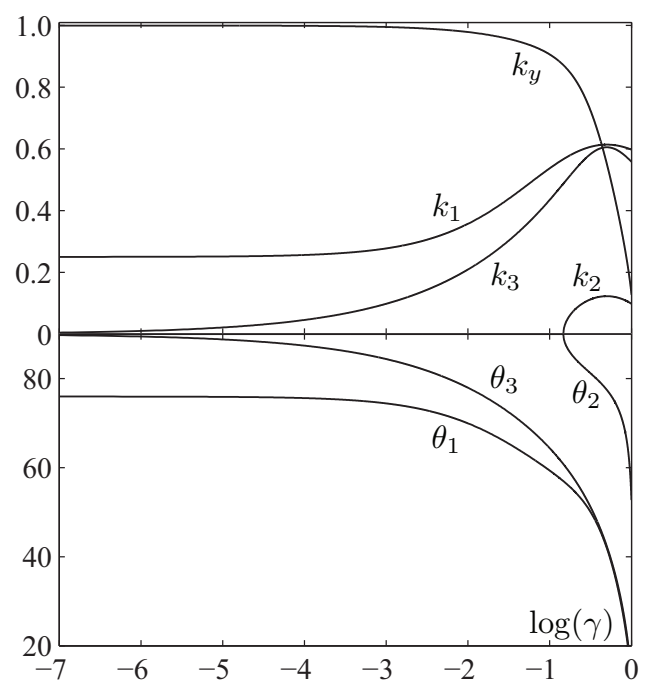

FIG. 14. (Color online) Dependence on damping with Dirichlet boundary conditions and $d=2 \pi$. (a) Critical forcing value versus $\gamma$. The dashed curve shows the uniform forcing limit of Eq. (10). (b) Wavenumbers $k_{y}, k_{1}, k_{2}$, and $k_{3}$. Note that $k_{2}$ is zero (i.e., $\kappa_{2}$ is real) for $\gamma>0.147$. (c) $\theta_{1}=\arctan \left(k_{y} / k_{1}\right), \theta_{2}=\arctan \left(k_{y} / k_{2}\right)$, and $\theta_{3}=$ $\arctan \left(k_{y} / k_{3}\right)$. Here $\log (\gamma)$ denotes the common logarithm (base 10).

with $Z=\left(z_{1}, z_{2}, z_{3}, \bar{z}_{3}\right)^{\mathrm{T}}, W=\left(w_{1}, w_{2}, w_{3},-\bar{w}_{3}\right)^{\mathrm{T}}$, and $\Phi_{ \pm}$ defined by Eq. (17). Note that eigenvectors must respect the phase relationships inherent in the definitions of $Z$ and $W$, which themselves reflect the phase condition (16) and the analog for $w_{1}$ and $w_{2}$ :

$$
w_{1}=-\Phi_{+} \bar{w}_{1}, \quad w_{2}=-\Phi_{-} \bar{w}_{2} .
$$

The phase shift of $\pi$ (in the forcing function) is the only thing which distinguishes $Z$ from $W$. As discussed further in Sec. V, the problem is invariant under reflection about $x=L / 2$ (switching $z_{j}$ and $w_{j}$ ) combined with translation through a half period of the forcing, which corresponds to a phase shift of $\pi / 2$ for subharmonic waves. This symmetry is represented in Eq. (30) by the action $\rho:(Z, W) \rightarrow i(W, Z)$ or, equivalently,

$$
\rho:\left(z_{j}, w_{j}\right) \rightarrow i\left(w_{j}, z_{j}\right), \quad j=1,2,3,
$$

which generates the cyclic group $C_{4}$. One consequence of this symmetry is that zero eigenvalues of the homogeneous linear system (30) come in pairs and the center eigenspace is two-dimensional.

Finding solutions of the bounded problem requires determining when the determinant of the linear system (30) vanishes. In the limit $L \rightarrow \infty$ this condition reduces to $\operatorname{det}\left(\mathrm{A}_{\infty}\right)=0$ because $\Lambda_{L}$ is suppressed by the exponentially small $\varepsilon_{3}$ term, indicating the decoupling of the two distant 
sides. This decoupled solvability condition on $A_{\infty}$ reduces to Eq. (18), a purely real equation. However, no such simplification occurs for finite $L$. In the general case two parameters must be varied to make the determinant in Eq. (30) vanish, and (for fixed $\gamma$ ) subharmonic solutions exist only for a discrete set of $k_{y}$ values.

\section{Dirichlet boundary conditions}

When Dirichlet boundary conditions are applied, the expansion (20) applies in region I, and a reflected version in region III, while the unforced region II requires the same expression in Eq. (28b). Altogether, we have

$$
\begin{aligned}
p^{\mathrm{I}}(x) & =z_{1} \sinh \left(\kappa_{1} x\right)+z_{2} \sinh \left(\kappa_{2} x\right), \\
p^{\mathrm{II}}(x) & =z_{3} e^{\kappa_{3}(d-x)}+w_{3} e^{\kappa_{3}(x-L+d)}, \\
p^{\mathrm{III}}(x) & =w_{1} \sinh \left[\kappa_{1}(L-x)\right]+w_{2} \sinh \left[\kappa_{2}(L-x)\right],
\end{aligned}
$$

where $\kappa_{1}$ and $\kappa_{2}$ again satisfy Eq. (9) and $\kappa_{3}$ satisfies Eq. (8).

At $x=d$ and $x=L-d$ the continuity of $P(x)$ and $P^{\prime}(x)$ requires

$$
\begin{aligned}
& 0=\operatorname{sh}_{1} z_{1}+\operatorname{sh}_{2} z_{2}-z_{3}-\varepsilon_{3} w_{3}, \\
& 0=\kappa_{1} \operatorname{ch}_{1} z_{1}+\kappa_{2} \operatorname{ch}_{2} z_{2}+\kappa_{3} z_{3}-\kappa_{3} \varepsilon_{3} w_{3}, \\
& 0=\operatorname{sh}_{1} w_{1}+\operatorname{sh}_{2} w_{2}-w_{3}-\varepsilon_{3} z_{3}, \\
& 0=\kappa_{1} \operatorname{ch}_{1} w_{1}+\kappa_{2} \operatorname{ch}_{2} w_{2}+\kappa_{3} w_{3}-\kappa_{3} \varepsilon_{3} z_{3} .
\end{aligned}
$$

As in Sec. III A, the problem with Dirichlet boundary conditions is identical in form to the Neumann case, but with sinh and cosh interchanged. Solutions exist when the determinant of the following linear system vanishes:

$$
\left(\begin{array}{cc}
\mathrm{B}_{\infty} & \Lambda_{L} \\
\Lambda_{L} & \mathrm{~B}_{\infty}
\end{array}\right)\left(\begin{array}{c}
Z \\
W
\end{array}\right)=0
$$

where

$$
\mathrm{B}_{\infty}=\left(\begin{array}{cccc}
\mathrm{sh}_{1} & \mathrm{sh}_{2} & -1 & 0 \\
\kappa_{1} \operatorname{ch}_{1} & \kappa_{2} \operatorname{ch}_{2} & \kappa_{3} & 0 \\
\operatorname{sh}_{1} \bar{\Phi}_{+} & \operatorname{sh}_{2} \bar{\Phi}_{-} & 0 & -1 \\
\kappa_{1} \operatorname{ch}_{1} \bar{\Phi}_{+} & \kappa_{2} \operatorname{ch}_{2} \bar{\Phi}_{-} & 0 & \bar{\kappa}_{3}
\end{array}\right)
$$

and $\Lambda_{L}$ is given by Eq. (32).

In the limit $L \rightarrow \infty$ the solvability condition reduces to $\operatorname{det}\left(\mathrm{B}_{\infty}\right)=0$, which is equivalent to Eq. (24). With finite $L$ two parameters must be varied to locate a subharmonic solution.

\section{Results for bounded domain}

The discrete set of subharmonic solutions that remain when the domain is finite is represented in Fig. 15 for the case of Neumann boundary conditions, and in Fig. 16 for the case of Dirichlet boundary conditions. These should be compared to Fig. 9 and Fig. 10, respectively. At each solution point in the $\left(k_{y}, f\right)$ plane there is a two-dimensional eigenspace that includes both one-sided and two-sided patterns. Examples of these two types of solutions at the lowest solution point of Fig. 15 are shown in Fig. 17. We note that most solutions for $k_{y}>1$ are not shown in Figs. 15 and 16 due to the numerical difficulty of resolving the roots of the determinant over this range. In fact, the solutions for $k_{y}>1$ grow increasingly dense and lie extremely close to the neutral stability curve of the semi-infinite case.

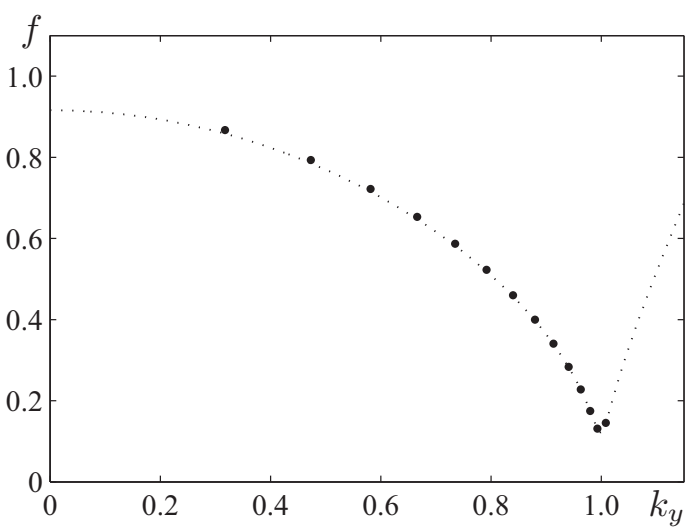

FIG. 15. Location of unmodulated subharmonic solutions with $d=0.4 \pi, \gamma=0.01, L=15 \pi$, and Neumann boundary conditions at $x=0, L$. The dotted curve shows the neutral stability curve in the semi-infinite case of Fig. 9. Most solutions for $k_{y}>1$ are not shown.

The set of unmodulated subharmonic solutions does not represent the full range of behavior permitted by Eq. (5). Pure subharmonic solutions require particular values of $k_{y}$ for fixed $\gamma, d$, and $L$ or, equivalently, particular values of $L$ for fixed $\gamma$, $d, k_{y}$. This can be seen as a resonance condition on $P(x)$ arising from interaction with the opposite side of the domain. Since pure subharmonic solutions are not possible for all values of $k_{y}$ and $L$ we consider modulated (quasiperiodic) solutions in the following section.

\section{MODULATED PATTERNS}

Equation (7) is generalized by introducing a detuning parameter and substituting

$h(\boldsymbol{x}, t)=\cos \left(k_{y} y\right)\left[\left(P_{+}(x) e^{i \delta t}+\bar{P}_{-}(x) e^{-i \delta t}\right) e^{i t}+\right.$ c.c. $]+\cdots$.

into Eq. (5). This is equivalent to looking for a Hopf bifurcation in the full nonlinear system (2). Again, only periodic profiles in the crosswise $y$ direction are considered. Collecting terms

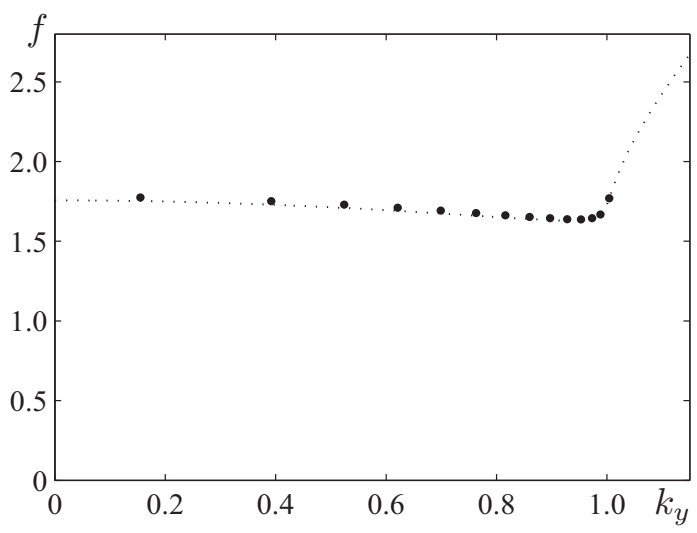

FIG. 16. Location of unmodulated subharmonic solutions with $d=0.4 \pi, \gamma=0.01, L=15 \pi$, and Dirichlet boundary conditions at $x=0, L$. The dotted curve shows the neutral stability curve in the semi-infinite case of Fig. 10. Most solutions for $k_{y}>1$ are not shown. 
(a)

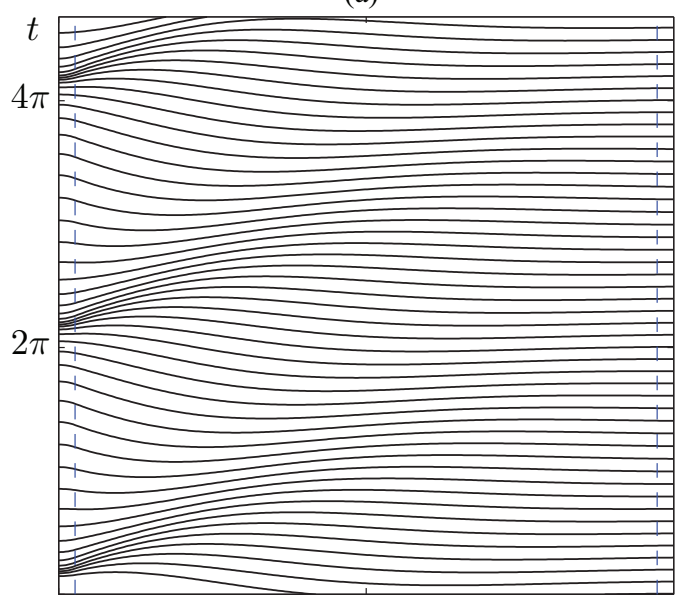

(b)

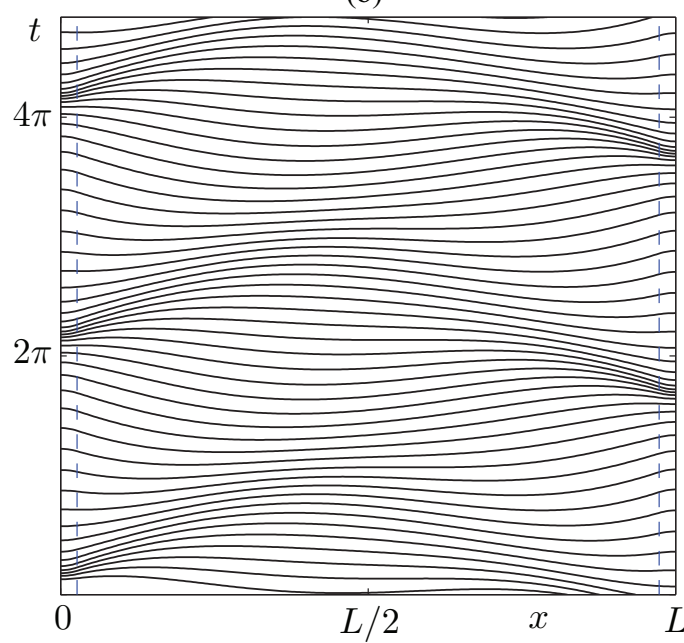

FIG. 17. (Color online) Spacetime plots of two different eigenfunctions for the lowest solution point in Fig. 15 at $\left(k_{y}, f\right)=$ (0.993250.13101). The eigenfunction in (a) is concentrated near $x=$ 0 while that in (b) describes balanced excitation that is out-of-phase (by $90^{\circ}$ ) at each side. Dashed lines show the edge of the forced regions.

leads to the ordinary differential equations:

$$
\left(1 \pm 2 i \gamma \omega_{ \pm}\right) P_{ \pm}^{\prime \prime}+\left[\omega_{ \pm}^{2}-k_{y}^{2}\left(1 \pm 2 i \gamma \omega_{ \pm}\right)\right] P_{ \pm}=f g(x) P_{\mp},
$$

where $\omega_{ \pm}=1 \pm \delta$.

In this section we take $g(x)$ to be the piecewise continuous antisymmetric function defined by Eq. (27) and, for simplicity, consider only Neumann boundary conditions at $x=0, L$; the treatment of Dirichlet boundary conditions is analogous (see Sec. III C2). The expansions appropriate to regions I-III take the same form as Eq. (28), namely,

$$
\begin{aligned}
P_{ \pm}^{\mathrm{I}}(x) & =z_{1}^{ \pm} \cosh \left(\kappa_{1} x\right)+z_{2}^{ \pm} \cosh \left(\kappa_{2} x\right), \\
P_{ \pm}^{\mathrm{II}}(x) & =z_{3}^{ \pm} e^{\kappa_{ \pm}(d-x)}+w_{3}^{ \pm} e^{\kappa_{ \pm}(x-L+d)} \\
P_{ \pm}^{\mathrm{III}}(x) & =w_{1}^{ \pm} \cosh \left[\kappa_{1}(L-x)\right]+w_{2}^{ \pm} \cosh \left[\kappa_{2}(L-x)\right] .
\end{aligned}
$$

Here $\kappa_{1}^{2}$ and $\kappa_{2}^{2}$ are the two roots of the quadratic polynomial

$$
a(\gamma, \delta) \kappa^{4}+b\left(\gamma, \delta, k_{y}\right) \kappa^{2}+c\left(\gamma, \delta, k_{y}\right)=0,
$$

where

$$
\begin{aligned}
& a=1+4 \gamma^{2} \omega_{+} \omega_{-}+4 i \gamma \delta, \\
& b=2\left[2-(1+2 i \gamma \delta) \omega_{+} \omega_{-}\right]-2 k_{y}^{2} a, \\
& c=\omega_{+}^{2} \omega_{-}^{2}-2 k_{y}^{2}(1+2 i \gamma \delta)+k_{y}^{4}\left(1+4 \gamma^{2}+8 \gamma^{2} \delta\right)-f^{2} .
\end{aligned}
$$

In contrast to the case of unmodulated solutions in Sec. III, these roots cannot be assumed to be real; the wavenumbers $\kappa_{1}$ and $\kappa_{2}$ will, in general, be complex. The wavenumbers $\kappa_{ \pm}$in region II (unforced) are defined by

$$
\kappa_{ \pm}^{2}=k_{y}^{2}-\frac{\omega_{ \pm}^{2}}{1 \pm 2 i \gamma \omega_{ \pm}},
$$

taking the root with positive real part.

Continuity conditions for $P_{ \pm}(x)$ and $P_{ \pm}^{\prime}(x)$ at $x=d$ and $x=L-d$ provide eight equations that, after using Eq. (40) to eliminate $z_{j}^{-}$and $w_{j}^{-}, j=1,2$, can be written in the manner of Eq. (30):

$$
\left(\begin{array}{cc}
\mathrm{A}_{\infty}^{\delta} & \Lambda_{L}^{\delta} \\
\Lambda_{L}^{\delta} & \mathrm{A}_{\infty}^{\delta}
\end{array}\right)\left(\begin{array}{c}
Z \\
W
\end{array}\right)=0
$$

where

$$
\begin{gathered}
\mathrm{A}_{\infty}^{\delta}=\left(\begin{array}{cccc}
\mathrm{ch}_{1} & \mathrm{ch}_{2} & -1 & 0 \\
\kappa_{1} \mathrm{sh}_{1} & \kappa_{2} \mathrm{sh}_{2} & \kappa_{+} & 0 \\
\operatorname{ch}_{1} \Psi\left(\kappa_{1}\right) & \operatorname{ch}_{2} \Psi\left(\kappa_{2}\right) & 0 & -1 \\
\kappa_{1} \operatorname{sh}_{1} \Psi\left(\kappa_{1}\right) & \kappa_{2} \operatorname{sh}_{2} \Psi\left(\kappa_{2}\right) & 0 & \kappa_{-}
\end{array}\right), \\
\Lambda_{L}^{\delta}=\left(\begin{array}{cccc}
0 & 0 & -\varepsilon_{+} & 0 \\
0 & 0 & -\kappa_{+} \varepsilon_{+} & 0 \\
0 & 0 & 0 & \varepsilon_{-} \\
0 & 0 & 0 & \kappa_{-} \varepsilon_{-}
\end{array}\right) .
\end{gathered}
$$

Here $Z=\left(z_{1}^{+}, z_{2}^{+}, z_{3}^{+}, z_{3}^{-}\right)^{\mathrm{T}}, W=\left(w_{1}^{+}, w_{2}^{+}, w_{3}^{+},-w_{3}^{-}\right)^{\mathrm{T}}, \kappa_{1}$ and $\kappa_{2}$ are defined by Eq. (42), $\kappa_{ \pm}$is given by Eq. (43), and $\varepsilon_{ \pm} \equiv \exp \left\{\kappa_{ \pm}(2 d-L)\right\}$. The factor $\Psi(\kappa)$ is given by

$$
\Psi(\kappa)=\frac{f}{\left(1-2 i \gamma \omega_{-}\right) \kappa^{2}+\left[\omega_{-}^{2}-k_{y}^{2}\left(1-2 i \gamma \omega_{-}\right)\right]} .
$$

\section{A. Results}

The linear system (44) can have nontrivial solutions only when the associated complex determinant vanishes and, in general, this requires varying two parameters. We calculate solution curves in the $\left(k_{y}, f, \delta\right)$ parameter space for fixed $\gamma$, $d$, and $L$. These curves include unmodulated subharmonic solutions where they cross $\delta=0$ and thus contain the discrete set of unmodulated solutions found in Sec. III D. In addition to these are a large number of modulated solutions $(\delta \neq 0)$ as illustrated in Fig. 18. The modulated solutions take the form either of isolas or of open curves terminating at $k_{y}=0$. The resonant values of $k_{y}$, where unmodulated subharmonic solutions are possible, organize the oscillations of these neutral stability curves in the $\left(f, k_{y}\right)$ plane; larger values of $L$ lead to more resonant values, and thus more rapid oscillations. 


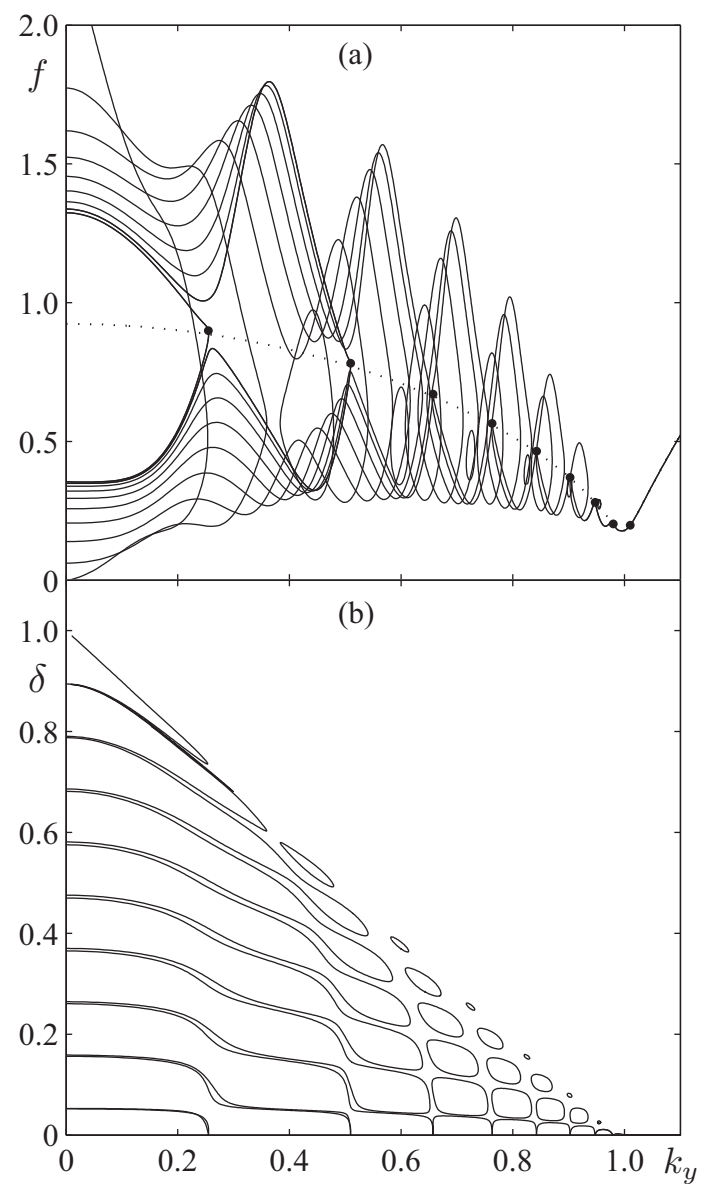

FIG. 18. Projection of the neutral stability curves defined by Eq. (44) with $\gamma=0.02, d=2 \pi / 5$, and $L=30$ onto (a) the $\left(k_{y}, f\right)$ plane and (b) the $\left(k_{y}, \delta\right)$ plane. Neumann boundary conditions are applied at $x=0, L$. The dotted curve in (a) shows the neutral stability curve in the semi-infinite case, and the solid dots mark the location of unmodulated solutions $(\delta=0)$. Solution branches with large $\delta$, including the one that approaches $\left(k_{y}, f\right)=(0,0)$ are associated with flat (long wavelength) solutions in Eq. (5).

The structure of these solution curves is clearest in the $(f, \delta)$ projection, where the region $k_{y}+\delta<1$ is largely filled by solution branches that seldom cross, and the order imposed by resonance conditions is apparent.

The qualitative nature of the solutions to Eq. (44) can be described in terms of their proximity to three limiting cases associated with the vertices of the triangular region $k_{y}+\delta<1$ in Fig. 18(b). Solutions near $k_{y}=1, \delta=0$ correspond to modulated crosswise oriented patterns. Solutions near $k_{y}=0$, $\delta=0$ correspond to modulated patterns oriented along the axis of vibration. Solutions with intermediate values of $k_{y}$ and $\delta \sim 0$ are obliquely oriented. The local minimum in Fig. 18(a) at $\left(k_{y}, f, \delta\right)=(0.99598,0.17771,0.0017482)$, for example, corresponds to a modulated crosswise oriented pattern. The associated eigenfunction oscillates rapidly in $y$, slowly in $x$, and has a temporal frequency near unity. Slow temporal modulations cause this onset pattern to alternate gradually from side to side with a period $\pi / \delta \simeq 1797$. At some points during this slow modulation the pattern is present in equal proportion at both endwalls and at other times the excitation is concentrated almost entirely near one wall. This type of solution, with small $\delta$, regardless of orientation, is expected to be representative of subharmonic patterns excited parametrically in finite containers, i.e., to persist in more realistic models.

In contrast to slowly modulated patterns with $\delta \ll 1$, solutions with large $\delta$ must be interpreted with caution. Equation (5) allows a constant solution $h(\boldsymbol{x}, t)=h_{0}$ with $f=0$ and this appears in Fig. 18 at $\left(k_{y}, f, \delta\right)=(0,0,1)$. Solutions near this point are composed of a large nearly constant component (related to this $h(\boldsymbol{x}, t)=h_{0}$ solution) and a small oscillatory component with frequency and wavenumber near 2 (i.e., harmonic waves). Such solutions, which are far from subharmonic waves, were not contemplated in Eq. (2) and, furthermore, violate conservation of volume if $h(\boldsymbol{x}, t)$ is taken as the surface deformation of a fluid confined to a finite domain. We do not expect the large $\delta$ solutions of Eq. (44) to be representative of more realistic parametrically forced systems where long wave excitations experience damping due to the container bottom, for example. Even if the solutions with large $\delta$ are excluded, however, the oscillatory resonant structure of Fig. 18 remains. Temporally modulated solutions that onset prior to nearby subharmonic modes [solid dots in Fig. 18(a)] are the predominant feature. In addition to temporal modulation, the eigenfunctions at the local minima of Fig. 18(a) exhibit varying spatial orientations. The proximity (with increasing forcing) of numerous modulated solutions can be expected to increase the complexity of the pattern selection process in the full (nonlinear) system even when the first pattern to onset is close to subharmonic cross-waves $\left(k_{y} \sim 1, \delta \ll 0\right)$.

The damping value of $\gamma=0.02$ used in Fig. 18 is relatively small. If $\gamma$ is decreased even further, the oscillations in the $\left(f, k_{y}\right)$ plane dip lower, and a few more isolas may appear, but the structure in the $(f, \delta)$ plane does not change much from Fig. 18(b). If $\gamma$ is increased, on the other hand, the oscillations stay closer to the curve defined by the semi-infinite case [the dotted curve in Fig. 18(a)] while open curves pinch off to create isolas that shrink and disappear, leaving a picture like that shown in Fig. 19 for $\gamma=0.1$. Note that two types of solutions are clearly separated in this figure. There is a continuous branch, with small Hopf frequency $\delta$, that oscillates near the neutral stability curve of the semi-infinite case and represents modulated subharmonic patterns. The solutions along this branch vary in orientation, being approximately crosswise near $k_{y}=1$ and dependent only on $x$ when $k_{y}=0$. The remaining solution curves, which are grouped near $k_{y}=0, \delta=1$, are qualitatively very distinct from subharmonic patterns, as discussed above.

As the width $d$ of the forced region is increased the neutral stability curves associated with additional oscillations in the forced region $x \leqslant d$ come into play (see Sec. IIIB) and complicate the picture shown in Fig. 18. The interaction of the first two such modes is shown in Fig. 20 for the width $d=\pi$. Note that it is the second neutral stability tongue (with reference to the semi-infinite case, shown as a dotted curve) which displays a greater number of associated solutions; a comparison with Fig. 18 suggests that its proximity reduces the number of additional modulated solutions associated with the primary mode. 


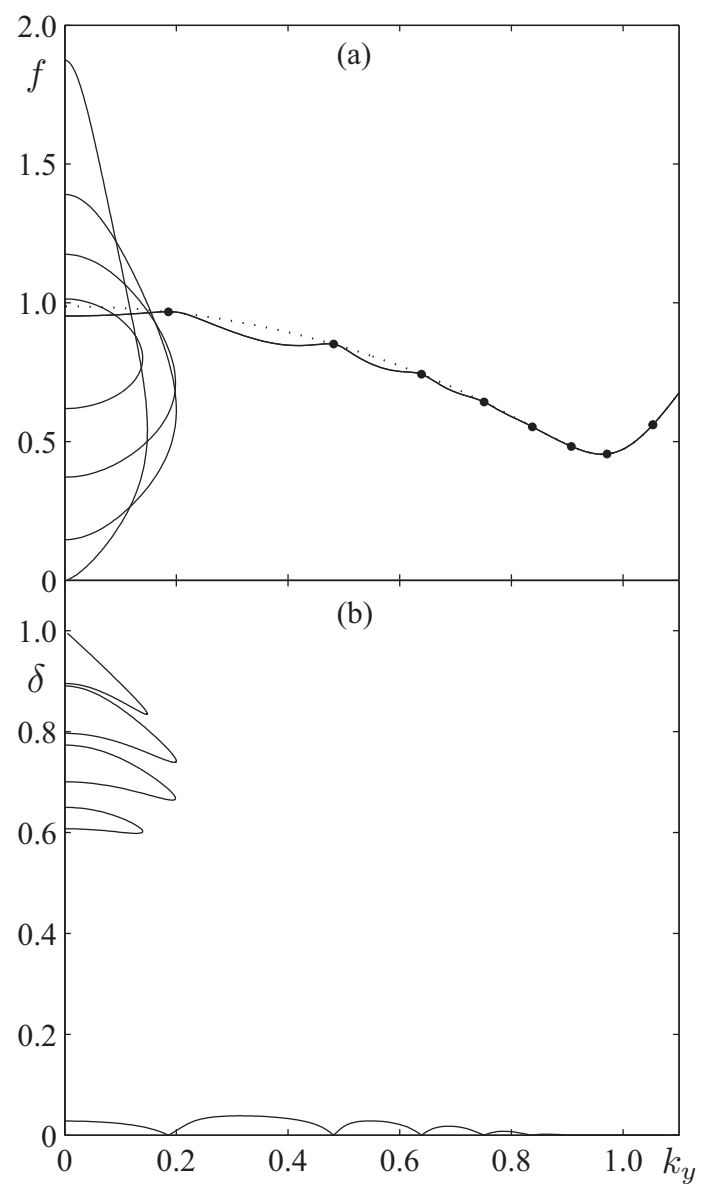

FIG. 19. Projection of the neutral stability curves defined by Eq. (44) with $\gamma=0.1, d=2 \pi / 5$, and $L=30$ onto (a) the $\left(k_{y}, f\right)$ plane and (b) the $\left(k_{y}, \delta\right)$ plane. Neumann boundary conditions are applied at $x=0, L$. The dotted curve in (a) shows the neutral stability curve in the semi-infinite case and the solid dots mark the location of unmodulated solutions $(\delta=0)$.

\section{WEAK INTERACTION: SYMMETRY CONSIDERATIONS}

The appearance of a Hopf bifurcation and, thus, temporally modulated (quasiperiodic) solutions is a generic feature of the (weak) interaction between two spatially separated subharmonic patterns driven by an antisymmetric forcing function like that of Eq. (27). The effect of the interaction between these two patterns, concentrated near opposing endwalls in the case of a horizontally vibrated container of fluid, can be understood as a symmetry-breaking perturbation (see, e.g., Refs. $[23,24]$ ) that reduces the symmetry group $D_{4}$ (describing the symmetries of a square) of the unperturbed (noninteracting) problem to the group $C_{4}$ (describing only rotations by $\pi / 2$ ).

First consider a container large enough that interaction between the opposing endwalls can be ignored. In that case, the eigenfunctions at onset correspond to those of the semi-infinite case (see Sec. III A) and, at opposite walls, can differ only by reflection in $x$ and a temporal phase (due to the antisymmetry of the applied forcing). The field $h(x, y, t)$ thus takes the form

$$
h=A_{1}(t) Z_{0}(x, y) e^{i t}+A_{2}(t) Z_{0}(L-x, y) e^{i t}+\text { c.c. }+\cdots,
$$

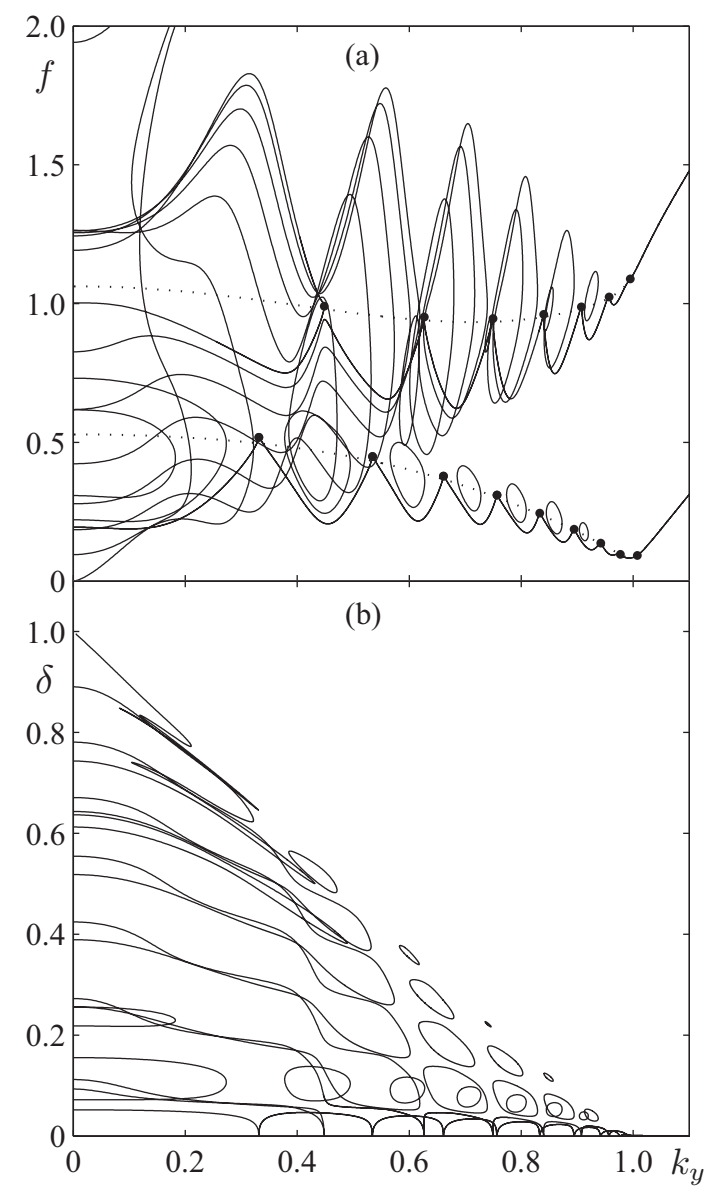

FIG. 20. Projection of the neutral stability curves defined by Eq. (44) with $\gamma=0.1, d=\pi$, and $L=30$ onto (a) the $\left(k_{y}, f\right)$ plane and (b) the $\left(k_{y}, \delta\right)$ plane. Neumann boundary conditions are applied at $x=0, L$. The dotted curves show the neutral stability curves in the semi-infinite case, and the solid dots mark the location of unmodulated solutions $(\delta=0)$.

where $Z_{0}(x, y)$ is a spatial eigenfunction of the semi-infinite problem, concentrated near $x=0$, and the slowly varying amplitudes $A_{1}$ and $A_{2}$ measure the strength of the pattern at each end of the container. The relevant spatiotemporal symmetry of this problem is a combined reflection in $x$ and time translation through one half period of the forcing: $x \rightarrow L-x, t \rightarrow t+\pi / 2$. This symmetry is represented by the action

$$
\rho:\left(A_{1}, A_{2}\right) \rightarrow i\left(A_{2}, A_{1}\right),
$$

which generates the group $C_{4}$. Note that the usual subharmonic (odd) symmetry is represented by $\rho^{2}:\left(A_{1}, A_{2}\right) \rightarrow-\left(A_{1}, A_{2}\right)$. The requirement of equivariance, and the absence of coupling between $A_{1}$ and $A_{2}$, dictates the form of the amplitude equations (truncated here at cubic order) describing dynamics near onset:

$$
\begin{aligned}
& \dot{A}_{1}=(-\gamma+i v) A_{1}+f \bar{A}_{1}-a\left|A_{1}\right|^{2} A_{1}, \\
& \dot{A}_{2}=(-\gamma+i v) A_{2}-f \bar{A}_{2}-a\left|A_{2}\right|^{2} A_{2} .
\end{aligned}
$$

Here $\gamma>0$ and $v$ are real damping and detuning parameters, respectively, $a=a_{r}+i a_{i}$ is a complex nonlinear coefficient, and $f$ can be taken as a real forcing amplitude after an 
appropriate rescaling and time shift. With no coupling, these parametrically forced Stuart-Landau equations are equivariant under the additional reflection, $R_{1}:\left(A_{1}, A_{2}\right) \rightarrow\left(-A_{1}, A_{2}\right)$, that acts only on $A_{1} ; R_{2}=\rho^{3} R_{1} \rho$ gives the corresponding reflection of $A_{2}$. The symmetry of the uncoupled problem is therefore $D_{4}$ rather than $C_{4}$. The primary bifurcation occurs when the larger of the eigenvalues of the trivial state, given by $\left.-\gamma \pm \sqrt{(} f^{2}-v^{2}\right)$ (each with algebraic multiplicity two), moves onto the positive real axis. This occurs when $f^{2}=\gamma^{2}+v^{2}$ and, consistent with the equivariant branching lemma (see, e.g., Ref. [25]), gives rise to eight solution branches via pitchfork bifurcation of the form $\pm(A, 0)$, $\pm(0, i A), \pm(A, i A)$, and $\pm(A,-i A)$. These primary branches bifurcate supercritically (subcritically) if the sign of $a_{r} \gamma-a_{i} v$ is positive (negative). In the supercritical case the $\pm(A, i A)$ and $\pm(A,-i A)$ branches are initially stable. Periodic solutions (modulated solutions of the full problem) can be generated only in secondary bifurcations.

A generic interaction between the two sides of the container breaks the symmetry of the unperturbed problem (50) and can be represented at leading order by a linear term (cf. [26]):

$$
\begin{aligned}
& \dot{A}_{1}=(-\gamma+i \nu) A_{1}+f \bar{A}_{1}-a\left|A_{1}\right|^{2} A_{1}+\epsilon A_{2}, \\
& \dot{A}_{2}=(-\gamma+i \nu) A_{2}-f \bar{A}_{2}-a\left|A_{2}\right|^{2} A_{2}+\epsilon A_{1},
\end{aligned}
$$

with $\epsilon=\epsilon_{r}+i \epsilon_{i},|\epsilon| \ll 1$. The eigenvalues of the trivial state are now given by $\left.-\gamma \pm i \epsilon_{i}+\sqrt{(} f^{2}-\nu^{2}+\epsilon_{r}^{2} \pm 2 i \nu \epsilon_{r}\right)$ and $\left.-\gamma \pm i \epsilon_{i}-\sqrt{(} f^{2}-v^{2}+\epsilon_{r}^{2} \pm 2 i v \epsilon_{r}\right)$, which are, in general, complex. If the interaction is conservative, for example, with $\epsilon_{r}=0$, then the bifurcation condition $f^{2}=\gamma^{2}+v^{2}$ still holds, but now signifies a Hopf bifurcation with frequency $\epsilon_{i}$. If $|\epsilon|$ is small, this primary branch of modulated solutions will not persist far from onset; at an $O(|\epsilon|)$ distance beyond the initial Hopf bifurcation the influence of the symmetry-breaking term diminishes and the modulated branch must be destroyed (in an infinite period saddle-node bifurcation, for example). Beyond this small interval of forcing values the unmodulated solution branches (perturbed versions of $\pm(A, i A)$ and so on) will again dominate.

These general symmetry considerations suggest that the subharmonic patterns exited by an isolated parametric forcing mechanism, like the endwall of a long horizontally vibrated container, will give way to modulated patterns when weak coupling to an identical but phase-shifted pattern, such as that at an opposing endwall, is taken into account. However, both the modulation frequency and the interval over which this modulated pattern exists will be small when the coupling is weak.

\section{DISCUSSION}

We have introduced and performed the linear analysis of a partial differential equation that generalizes Mathieu's equation and is therefore relevant to a range of parametrically forced pattern forming systems. We focused on an out-of-phase forcing mechanism concentrated near opposite boundaries, which naturally arises for back-and-forth or rocking motion. The experiment of direct interest here is the cross-wave instability in a horizontally vibrated container of fluid. In that case the localized forcing mechanism has a characteristic width $d$ set by the depth of the fluid [27]. This forcing is approximated by a piecewise constant function in order to obtain analytical solutions. The detailed solutions obtained allow one to clearly see how the properties of the critical eigenfunctions including onset value, orientation, and modulation rate (both spatial and temporal) depend on the important parameters of the problem: spatial detuning (crosswise wavenumber), forcing width, damping, boundary conditions, and container length. This dependence can be quite difficult to measure in an experiment [9]. The generalized Mathieu equation considered here should therefore be viewed as a complement to experiments or to more rigorous models that require numerical solution. Many features of the model described here are consistent with experiments, while others suggest new regimes of interest.

With respect to cross-wave experiments, the generalized Mathieu equation strongly suggests that, neglecting the effect of spatial detuning, pure cross-waves (oriented at $90^{\circ}$ with respect to the axis of vibration) can be observed only in the limit of small damping. This is consistent with their observation in low frequency wavemaker experiments in water [14,17-19,21,22,28,29] and the emergence of oblique waves in certain higher frequency experiments [10,11] and with silicone oil [9]. Boundary conditions also play a critical role. With Neumann boundary conditions, the limit of small damping is sufficient to obtain pure cross-waves. This is not so with Dirichlet boundary conditions where the wavenumber $k_{1}$ characterizing oscillations in the forced region remains finite unless the width $d$ of that region becomes large.

Boundary conditions are most important when the forced region is narrow, as expected. The critical onset value always increases with decreasing forcing width, but does so more rapidly with Dirichlet than with Neumann boundary conditions. Furthermore, when the forcing is narrow, Dirichlet boundary conditions lead to a more rotated pattern at onset.

Another feature that finds support in experimental observations of cross-waves [29] is the transition from standing to traveling waves. With the simple piecewise constant approximation used here this transition occurs abruptly at the edge of the forced region. Continuity conditions at this point are crucial in determining both the critical forcing value and the wavenumbers that characterize the orientation and modulation of the pattern. If the forcing is treated more realistically, this transition will be gradual. Nonetheless, the need for the pattern to cover both a supercritical (standing wave) domain and a subcritical (traveling wave) domain is also expected to play an important role in the selection process in more realistic models.

Multiplicity of solutions is a prominent feature of the model analyzed here, and one that is consistent with many crosswave experiments $[14,17,18,22,29,30]$. In the semi-infinite case there is a family of solutions parameterized by $k_{y}$, the crosswise wavenumber. Although, for most parameters, patterns with $k_{y} \simeq 1$ are selected at onset, this linear selection need not be unambiguous or strong. If Dirichlet boundary conditions are used with narrow forcing, for example, then rapid oscillations in $x$ (see Fig. 10) produce a visibly rotated pattern. It is important to note that, in the case of large-aspectratio systems where $k_{y}$ can take nearly any value, even if crosswise oriented patterns are initially selected, the presence of additional, weakly damped, obliquely oriented modes may have a significant effect on dynamics above onset. 
In addition to variable orientation, there are a series of nested neutral stability tongues (Fig. 1) associated with increasing (half) oscillations within the forced region. This multiplicity results directly from the finite width of the forcing and is therefore absent in the standard NLS models describing cross-waves [12-15]. The importance of this type of multiplicity increases with the extent of the forced region.

Finally, a great deal of complexity is related to the temporally modulated (quasiperiodic) solutions that arise via Hopf bifurcation when the domain is finite in length. These modulated solutions can be understood as the result of symmetrybreaking due to weak interaction between waves coming from opposing sides, and describe patterns of excitation that alternate slowly from one side to the other. Furthermore, if the damping is small, this modulated solution (for a given $k_{y}$ ) need not be unique. Closed solution curves (isolas) describing more rapidly modulated patterns appear as in Fig. 18. These may onset before the more weakly modulated subharmonic patterns, although solutions with large modulation frequency must be viewed skeptically within the generalized Mathieu equation model. So far, modulated solutions that alternate from side to side have not been observed experimentally, probably owing to the small forcing interval over which they persist. These interesting solutions should be observable, however, when the interaction of the two sides is large enough, as with relatively short containers and small viscosity.

\section{ACKNOWLEDGMENT}

This work was supported by the Spanish Ministry of Science and Innovation (currently, Ministry of Economy and Competitiveness) under project AYA2010-19081.
[1] V. I. Arnold, Mathematical Methods of Classical Mechanics, Graduate Texts in Mathematics Vol 60 (Springer-Verlag, New York and Berlin, 1978).

[2] M. Faraday, Philos. Trans. R. Soc. Lond. 121, 299 (1831).

[3] J. Miles and D. Henderson, Annu. Rev. Fluid Mech. 22, 143 (1990).

[4] T. B. Benjamin and F. Ursell, Proc. R. Soc. Lond. A 225, 505 (1954).

[5] A. C. Skeldon and J. Porter, Phys. Rev. E 84, 016209 (2011).

[6] W. I. Newman, R. H. Rand, and A. L. Newman, Chaos 9, 242 (1999).

[7] D. Armbruster, M. George, and I. Oprea, Chaos 11, 52 (2001).

[8] T.-C. Jo and D. Armbruster, Phys. Rev. E 68, 016213 (2003).

[9] J. Porter, I. Tinao, A. Laverón-Simavilla, and C. A. Lopez, Fluid Dyn. Res. 44, 065501 (2012).

[10] S. Taneda, Fluid Dyn. Res. 13, 119 (1994).

[11] F. Moisy, G.-J. Michon, M. Rabaud, and E. Sultan, Phys. Fluids 24, 022110 (2012).

[12] S. Lichter and J. Chen, J. Fluid Mech. 183, 451 (1987).

[13] A. J. Bernoff, L. P. Kwok, and S. Lichter, Phys. Fluids A 1, 678 (1989).

[14] L. Shemer and E. Kit, J. Fluid Mech. 209, 249 (1989).
[15] A. F. Jones, J. Fluid Mech. 138, 53 (1984).

[16] W. Zhang and J. Viñals, J. Fluid Mech. 336, 301 (1997).

[17] B. J. S. Barnard and W. G. Pritchard, J. Fluid Mech. 55, 245 (1972).

[18] S. Lichter and W. B. Underhill, Phys. Rev. A 35, 5282 (1987).

[19] L. Shemer and S. Lichter, Phys. Fluids 30, 3427 (1987).

[20] S. Lichter and A. J. Bernoff, Phys. Rev. A 37, 1663 (1988).

[21] L. Shemer and S. Lichter, Exp. Fluids 9, 148 (1990).

[22] W. B. Underhill, S. Lichter, and A. J. Bernoff, J. Fluid Mech. 225, 371 (1991).

[23] M. Golubitsky and D. G. Shaeffer, Singularities and Groups in Bifurcation Theory, Vol. 1 (Springer, New York, 1985).

[24] J. D. Crawford and E. Knobloch, Annu. Rev. Fluid Mech. 23, 341 (1991).

[25] R. Hoyle, Pattern Formation: An Introduction to Methods (Cambridge University Press, Cambridge, 2006).

[26] J. Moehlis and E. Knobloch, Physica D 135, 263 (2000).

[27] F. Varas and J. M. Vega, J. Fluid Mech. 579, 271 (2007).

[28] J. D. Lin and L. N. Howard, Hydrodynamics Laboratory Technical Report 44, MIT (1960).

[29] S. Lichter and L. Shemer, Phys. Fluids 29, 3971 (1986).

[30] H. Ayanle, A. J. Bernoff, and S. Lichter, Physica D 43, 87 (1990). 